\title{
Lyapunov Functions for Multimachine Power Systems with Dynamic Loads
}

\author{
Robert J. Davy and Ian A. Hiskens, Senior Member, IEEE
}

\begin{abstract}
This paper develops Lyapunov functions for power systems which have dynamic reactive power loads. These functions are extensions of earlier Lyapunov functions derived for structure preserving power system models having static loads. In order to obtain strict Lyapunov functions, the transient response of the dynamic load must have a logarithmic dependence on voltage. A Lyapunov function is initially generated using a "first integral" analysis. A Popov criterion analysis is then undertaken for comparison. The developed Lyapunov functions enable the investigation of the interaction between generator (angle) and load dynamics in multimachine power systems.
\end{abstract}

Index Terms - Load dynamics, Lyapunov functions, power system stability.

\section{INTRODUCTION}

$\mathbf{S}$ IGNIFICANT progress has been made over the last few decades in the development and use of Lyapunov (energy) functions for multimachine power systems [11], [30], [31]. Energy functions have been proposed for power system models which include the full network topology [14], [26], [27], constant real power and static voltage dependent reactive power loads [20], higher order generator models [2], [28], and FACTS devices [9], [22], [29]. The focus of these developments has been the assessment of generator (angle) stability, i.e., determining whether all generators in the system remain in synchronism following a disturbance.

Energy functions provide a direct approach to such assessment. First, a "critical" value of energy is determined [3], [4]. This critical value provides an estimate of the maximum amount of energy that can be gained by the system during a disturbance without the system losing stability. If the system acquires less energy, stability will be guaranteed. However if the system acquires a greater amount of energy, it may be unstable. Stability assessment therefore reduces to a comparison of the critical energy with the energy of the system at the beginning of the postdisturbance period. The advantage of energy function methods is that stability can be determined without the need to obtain the system's postdisturbance trajectory.

Manuscript received June 8, 1995; revised February 17, 1997. This work was supported by the Australian Research Council through the Grant "Power System Security Analysis and Enhancement" and the Australian Electricity Supply Industry Research Board through the Grant "Voltage Collapse Analysis and Control." This paper was recommended by Associate Editor M. Ilic.

R. J. Davy is with Honeywell Limited, Adamstown, NSW 2289 Australia.

I. A. Hiskens is with the Department of Electrical and Computer Engineering, The University of Newcastle, Callaghan, NSW 2308 Australia (e-mail: ianh@ee.newcastle.edu.au).

Publisher Item Identifier S 1057-7122(97)06578-1.
While energy function techniques have been shown to be useful for angle stability assessment, little work has been undertaken in the development of energy function ideas for voltage stability (voltage collapse) assessment. Generically, the voltage collapse process involves an initiating disturbance which reduces the ability of the power system to supply the load demand. Voltages fall, causing an initial reduction in load demand. However load demand subsequently (at least partially) recovers, leading to further overloading of the weakened system, and further depression of voltages. Hence the voltage collapse process is driven largely by the dynamics of load recovery, rather than generator dynamics. Therefore energy functions must capture these extra dynamics.

Some initial ideas for energy functions of load systems were proposed in [18] and [19]. However these energy functions did not include generator dynamics. As explained in [21], while voltage collapse may be largely load driven, there is often some interaction between angle and voltage dynamics. This paper therefore proposes an energy function which takes account of both generator and load dynamics. One of the important features of voltage collapse is the limiting of reactive power which can be supplied from generators and other devices such as static var compensators (SVC's). It is shown in [22] how this limiting can be rigorously incorporated into energy functions. Those ideas are directly applicable to the energy functions proposed here.

Lyapunov functions for multimachine systems (without load dynamics) have been obtained through the use of a number of techniques. A first integral approach has been used for undamped systems, see for example, [5], [31], [32]. This is a somewhat ad hoc method, but has yielded some useful results. A more general approach to establishing Lyapunov functions is based on the multivariable Popov criterion. This approach follows from work of Anderson [1], and establishes Lyapunov stability for linear systems with nonlinear feedback. The resulting Lyapunov function has the Luré-Postnikov form. This method was used in a power systems context by Willems for the reduced network model [34], and by Hill and others for the structure preserving model [12], [14].

The paper is structured as follows. Section II provides background to the structure preserving power system model. The center of angle (COA) formulation is developed. The dynamic load model is established. First integral analysis is undertaken in Section III to obtain a Lyapunov function. The Popov criterion in then used in Section IV to provide a systematic development of the energy function. Section V uses the Popov criterion to establish energy functions for 
the machine reference angle model of the power system. Connections to gradient system ideas are made in Section VI. Also an extension to the dynamic load model is considered. Examples are presented in Section VII. Conclusions are drawn in Section VIII.

\section{MODELING}

\section{A. System Structure}

The usual assumptions underlying the structure preserving model of the power system shall be made for convenience. The first of those assumptions is the use of the classical machine model for synchronous machines. Such machines are therefore represented by a constant voltage in series with the transient reactance.

Let the power system consist of $n_{0}$ buses, with generators attached to $m$ of the buses. Hence there are $n_{0}-m$ load buses with no generation. The power system is augmented by $m$ fictitious buses representing the generator internal buses. The total number of buses in the augmented network is therefore $n_{0}+m:=n$.

The network is assumed to be lossless, so all lines are modeled as series reactances. The bus admittance matrix $Y$ is therefore purely imaginary, with elements $Y_{i j}=j B_{i j}$.

Let the complex voltage at the $i$ th bus be the (time varying) phasor $V_{i} \angle \delta_{i}$ where $\delta_{i}$ is the bus phase angle with respect to a synchronously rotating reference frame. The bus frequency deviation is given by $\omega_{i}=\dot{\delta}_{i}$. Define

$$
\begin{aligned}
\underline{\delta} & =\left[\delta_{1}, \cdots, \delta_{n}\right]^{t} \\
\underline{\omega}_{g} & =\left[\omega_{n_{0}+1}, \cdots, \omega_{n}\right]^{t} \\
\underline{V} & =\left[V_{1}, \cdots, V_{n}\right]^{t}
\end{aligned}
$$

where $V_{i}=E_{i}$ for $i=n_{0}+1, \cdots, n$ with $E_{i}$ being the (constant) voltage magnitude of the internal generator bus.

Let $P_{b_{i}}$ and $Q_{b_{i}}$ denote the total real and reactive power leaving the $i$ th bus via transmission lines. Then

$$
\begin{aligned}
& P_{b_{i}}(\underline{\delta}, \underline{V})=\sum_{j=1}^{n} V_{i} V_{j} B_{i j} \sin \left(\delta_{i}-\delta_{j}\right) \\
& Q_{b_{i}}(\underline{\delta}, \underline{V})=-\sum_{j=1}^{n} V_{i} V_{j} B_{i j} \cos \left(\delta_{i}-\delta_{j}\right) .
\end{aligned}
$$

Define

$$
\begin{aligned}
& \underline{P}_{b}=\left[P_{b_{1}}, \cdots, P_{b_{n}}\right]^{t}=\left[\underline{P}_{l}^{t} \underline{P}_{g}^{t}\right]^{t} \\
& \underline{Q}_{b}=\left[Q_{b_{1}}, \cdots, Q_{b_{n}}\right]^{t}=\left[\underline{Q}_{l}^{t} \underline{Q}_{g}^{t}\right]^{t}
\end{aligned}
$$

where subscripts $l$ and $g$ refer to $n_{0}$-vectors and $m$-vectors, respectively.

Synchronous machines are assumed to obey the usual swing equations

$$
M_{i} \dot{\omega}_{g_{i}}+D_{g_{i}} \omega_{g_{i}}+P_{b_{i+n_{0}}}(\underline{\delta}, \underline{V})=P_{M_{i}}
$$

where $M_{i}, D_{g_{i}}, P_{M_{i}}$ are the machine inertia, damping constant and mechanical power, respectively. In the center of angle analysis it will be assumed that all machine damping constants are zero. However damping will be allowed in the analysis of the machine reference angle model.
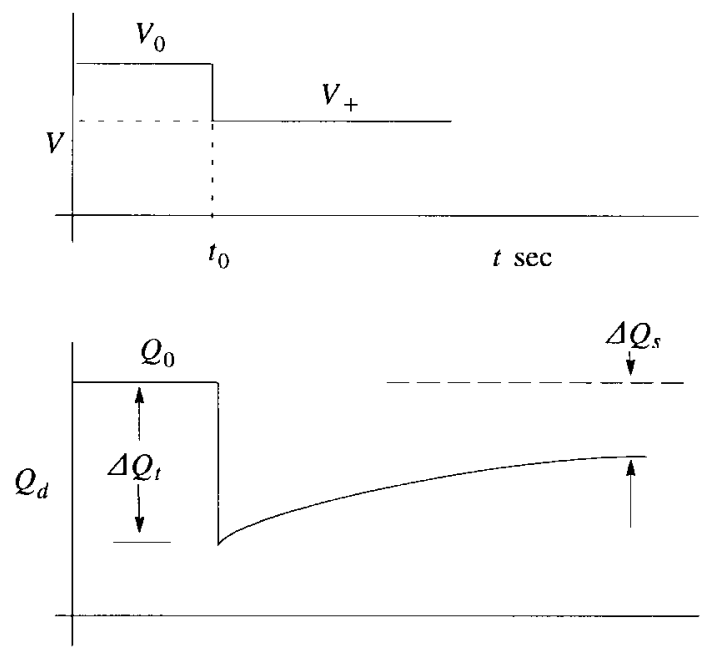

Fig. 1. Generic load response to a step in voltage.

\section{B. Load Modeling}

In the development of strict Lyapunov functions of the form commonly used in power system analysis, it is necessary to assume real power demand is composed of a constant component and a frequency dependent component [14]. Therefore we shall assume real power demand is given by

$$
P_{d_{i}}\left(\omega_{i}\right)=P_{d_{i}}^{0}+\omega_{i} D_{l_{i}} \quad i=1, \cdots, n_{0} .
$$

Fig. 1 shows the generic behavior of aggregate reactive power demand in response to a step change in voltage. It has been observed [25] and analytically justified [15] that in response to a step reduction in voltage, load demand falls quickly, then recovers to a new steady-state value. The aim of this paper is to develop Lyapunov functions which capture that form of response. A model for this dynamic recovery behavior was proposed in [15]

$$
\begin{aligned}
T_{q_{i}} \dot{x}_{q_{i}} & =-x_{q_{i}}+Q_{s_{i}}^{0}-Q_{t_{i}}\left(V_{i}\right) \\
Q_{d_{i}}\left(x_{q_{i}}, V_{i}\right) & =x_{q_{i}}+Q_{t_{i}}\left(V_{i}\right)
\end{aligned}
$$

for $i=1, \cdots, n_{0}-m$. This model gives a steady-state reactive power demand of $Q_{d_{i}}=Q_{s_{i}}^{0}$. We shall assume for now that $Q_{s_{i}}^{0}$ is a constant. This makes the analysis clearer. However this assumption is relaxed in Section VI-B, where we show that $Q_{s_{i}}$ can be voltage dependent.

The initial transient step change in the reactive power demand is described by $Q_{t_{i}}\left(V_{i}\right)$. Typically this transient load characteristic is a polynomial or exponential function of voltage. However to establish Lyapunov functions, $Q_{t_{i}}\left(V_{i}\right)$ must take the form

$$
Q_{t_{i}}\left(V_{i}\right)=Q_{t_{i}}^{0} \ln \left(\frac{V_{i}}{\mu_{i}}\right)
$$

where $Q_{t_{i}}^{0}$ and $\mu_{i}$ are positive constants. Note though that the ability to choose $Q_{t_{i}}^{0}$ and $\mu_{i}$ independently means that 'normal' voltage dependent functions can be approximated (at least locally) using this logarithmic form. To illustrate, consider a conventional transient load characteristic $Q_{t}(V)=0.4 V^{2}$. This can be locally approximated around the operating point $V=1.04 \mathrm{pu}$ by the logarithmic load characteristic $Q_{t}(V)=$ 


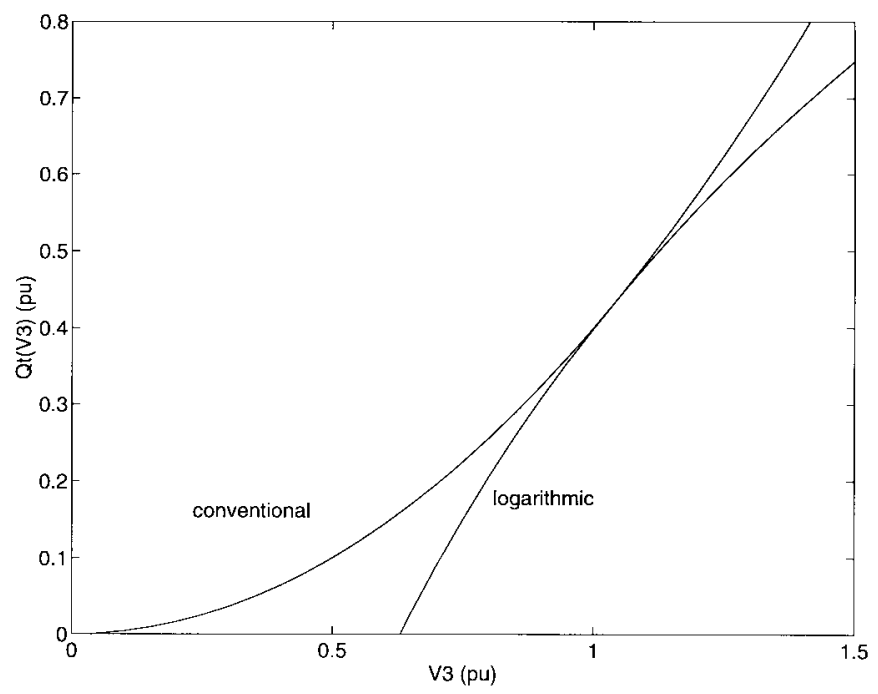

Fig. 2. Conventional transient load characteristic and a logarithmic approximation.

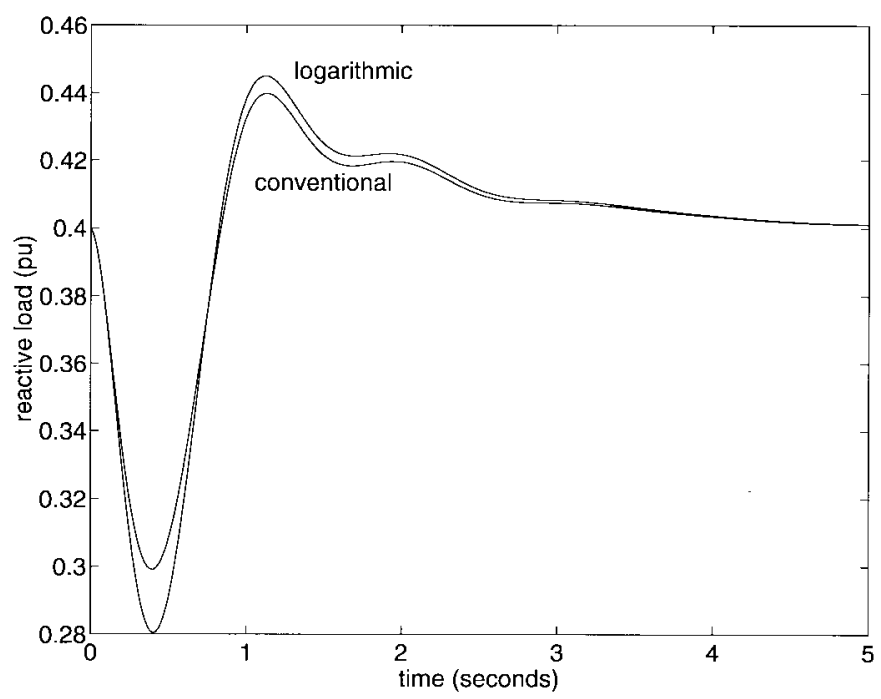

Fig. 3. Load reactive power for conventional and logarithmic characteristics following a large disturbance.

$0.8634 \ln \left(\frac{V}{0.6301}\right)$, i.e., $Q_{t}^{0} \approx 0.8634$ and $\mu \approx 0.6301$. Fig. 2 shows a comparison of the two load characteristics. Variations in reactive power load following a typical large disturbance are shown in Fig. 3.

For later analysis it is useful to define

$$
\gamma_{i}=\exp \left(\frac{Q_{s_{i}}^{0}}{Q_{t_{i}}^{0}}\right) \mu_{i}
$$

so that

$$
Q_{s_{i}}^{0}-Q_{t_{i}}\left(V_{i}\right)=-Q_{t_{i}}^{0} \ln \left(\frac{V_{i}}{\gamma_{i}}\right)
$$

Establishing power balance at the load buses gives the power flow equations

$$
\begin{array}{r}
P_{d_{i}}\left(\omega_{i}\right)+P_{b_{i}}(\underline{\delta}, \underline{V})=0 \\
Q_{d_{i}}\left(x_{q_{i}}, V_{i}\right)+Q_{b_{i}}(\underline{\delta}, \underline{V})=0
\end{array}
$$

for $i=1, \cdots, n_{0}$

\section{Center of Angle (COA) Formulation}

Power systems possess translational symmetry because bus phase angles $\delta_{i}$ are only defined relative to other phase angles. It is therefore convenient to establish a reference angle. Initially we shall use a weighted average angle, called the center-of-angle (COA), as the reference. In establishing this center of angle formulation, it is assumed that all generator and load damping is zero. In Section V-A, a machine angle reference formulation shall be developed. In that case, there is no restriction on damping.

The COA of an $m$-machine system is defined as

$$
\delta_{0}=\frac{1}{M_{T}} \sum_{i=1}^{m} M_{i} \delta_{i+n_{0}}
$$

where $M_{T}=\sum_{i=1}^{m} M_{i}$. It follows that

$$
\omega_{0}=\frac{1}{M_{T}} \sum_{i=1}^{m} M_{i} \omega_{g_{i}}, \quad \dot{\omega}_{0}=\frac{1}{M_{T}} \sum_{i=1}^{m} M_{i} \dot{\omega}_{g_{i}} .
$$

The machine rotor angles and bus phase angles referred to the COA are

$$
\theta_{i}=\delta_{i}-\delta_{0} \quad i=1, \cdots, n
$$

Define

$$
\underline{\theta}=\left[\theta_{1}, \cdots, \theta_{n}\right]^{t}=\left[\underline{\theta}_{l}^{t} \underline{\theta}_{g}^{t}\right]^{t}
$$

where subscripts $l, g$ refer to load and generator buses, respectively. Equation (14) gives

$$
\begin{aligned}
\dot{\theta}_{i} & =\dot{\delta}_{i}-\dot{\delta}_{0} \\
& =\omega_{i}-\omega_{0}:=\tilde{\omega}_{i}
\end{aligned}
$$

for $i=1, \cdots, n$. Summing the swing equations (3) over all machines (with $D_{g_{i}}=0$ ), and noting that $\sum_{i=1}^{n} P_{b_{i}}=0$ gives

$$
M_{T} \dot{\omega}_{0}=\left(\sum_{i=1}^{m} P_{M_{i}}-\sum_{i=1}^{n_{0}} P_{d_{i}}^{0}\right) \equiv P_{\mathrm{COA}} .
$$

It is common to assume that $P_{\mathrm{COA}}=0$. The swing equations (3) can then be written in the COA reference frame as

$$
\begin{aligned}
\dot{\theta}_{i+n_{0}} & =\tilde{\omega}_{g_{i}} \\
M_{i} \dot{\tilde{\omega}}_{g_{i}} & =P_{M_{i}}-P_{b_{i+n_{0}}}(\underline{\theta}, \underline{V})
\end{aligned}
$$

where $P_{b_{i}}(\underline{\theta}, \underline{V})$ is the COA form of (1). Similarly $Q_{b_{i}}(\underline{\theta}, \underline{V})$ refers to the COA form of (2).

\section{System Model}

Combining the machine swing equations with the load model and power balance equations gives the singularly perturbed form of the COA structure preserving model

$$
\begin{aligned}
\underline{\tilde{\omega}}_{g} & =-M_{g}^{-1}\left(\underline{P}_{g}(\underline{\theta}, \underline{V})-\underline{P}_{M}\right) \\
\underline{\dot{\theta}} & =R_{2} \underline{\tilde{\omega}}_{g}-R_{1} D_{l}^{-1}\left(\underline{P}_{l}(\underline{\theta}, \underline{V})+\underline{P}_{d}^{0}\right) \\
\underline{\dot{x}}_{q} & =-T_{q}^{-1} \underline{x}_{q}-T_{q}^{-1} Q_{t}^{0} \underline{k}(\underline{V}) \\
\underline{\dot{V}} & =-E^{-1}[V]^{-1}\left(\underline{Q}_{l}(\underline{\theta}, \underline{V})+\underline{Q}_{d}\left(\underline{x}_{q}, \underline{V}\right)\right)
\end{aligned}
$$


where

$$
\begin{aligned}
& M_{g}=\operatorname{diag}\left\{M_{i}\right\} \\
& D_{l}=\operatorname{diag}\left\{D_{l_{i}}\right\} \\
& T_{q}=\operatorname{diag}\left\{T_{q_{i}}\right\} \\
& Q_{t}^{0}=\operatorname{diag}\left\{Q_{t_{i}}^{0}\right\} \\
& E=\operatorname{diag}\left\{\epsilon_{i}\right\} \\
& {[V]=\operatorname{diag}\left\{V_{i}\right\}} \\
& R_{1}=\left[\begin{array}{c}
I_{n_{0}} \\
0_{m \times n_{0}}
\end{array}\right], \quad R_{2}=\left[\begin{array}{c}
0_{n_{0} \times m} \\
I_{m}
\end{array}\right] \\
& k_{i}\left(V_{i}\right)=\ln \left(\frac{V_{i}}{\gamma_{i}}\right) \\
& Q_{d_{i}}\left(\underline{x}_{q}, \underline{V}\right)= \begin{cases}x_{q_{i}}+Q_{t_{i}}^{0} \ln \left(\frac{V_{i}}{\mu_{i}}\right) & i=1, \cdots, n_{0}-m \\
0 & i=n_{0}-m+1, \cdots, n_{0}\end{cases}
\end{aligned}
$$

The equations are singularly perturbed through the introduction of the term $D_{l} \dot{\theta}_{i}$ into the real power balance equations for load buses, and the term $\epsilon_{i} \dot{V}_{i}$ into the reactive power balance equations for load buses. These singular perturbations are not required for the first integral analysis of Section III, and in that case are set to zero. However they are required for the later Popov criterion analysis, though they are set to zero in the final Lyapunov function. (Note that in a sense the $D_{l_{i}} \dot{\theta}_{i}$ term can be physically interpreted as load damping. However recall that the COA formulation required load damping to be zero. The machine angle reference formulation of Section $\mathrm{V}$ has no such requirement of zero load damping. Therefore in that formulation $D_{l_{i}} \dot{\theta}_{i}$ can be validly interpreted as load damping.)

For later analysis, it is convenient to define

$$
\underline{P}^{0}=\left[\begin{array}{ll}
-\underline{P}_{d}^{0 t} & \underline{P}_{M}^{t}
\end{array}\right]^{t}
$$

and vector functions

$$
\begin{aligned}
\underline{f}(\underline{\theta}, \underline{V}) & =\underline{P}_{b}(\underline{\theta}, \underline{V})-\underline{P}^{0} \\
& =\left[\begin{array}{l}
\underline{P}_{l}(\underline{\theta}, \underline{V})+\underline{P}_{d}^{0} \\
\underline{P}_{g}(\underline{\theta}, \underline{V})-\underline{P}_{M}
\end{array}\right]=\left[\begin{array}{l}
f \\
l
\end{array}(\underline{\underline{\theta}}, \underline{V})\right. \\
\underline{\underline{\theta}} & \underline{\underline{V}})] \\
\underline{g}\left(\underline{\theta}, \underline{x}_{q}, \underline{V}\right) & =[V]^{-1}\left(\underline{Q} \underline{Q}_{l}(\underline{\theta}, \underline{V})+\underline{Q}_{d}\left(\underline{x}_{q}, \underline{V}\right)\right) .
\end{aligned}
$$

\section{First Integral Analysis}

Our initial approach to constructing a Lyapunov function is to use first integral analysis. A clear outline of the first integral analysis is given in [31] and [32]. Basically the (nonsingularly perturbed) system equations are scaled appropriately and summed together. The procedure given in [31] and [32] will be followed here.

The first step is to multiply the reactive power balance equations (11) by $\frac{\dot{V}_{i}}{V_{i}}$, taking into account (2)

$Q_{d_{i}}\left(x_{q_{i}}, V_{i}\right) \frac{\dot{V}_{i}}{V_{i}}-B_{i i} \dot{V}_{i} V_{i}-\sum_{j=1, \neq i}^{n} B_{i j} \dot{V}_{i} V_{j} \cos \left(\theta_{i}-\theta_{j}\right)=0$.
Multiply the swing equations (16) by $\tilde{\omega}_{g_{i-n_{n}}}=\dot{\theta}_{i}$, taking account of (1)

$$
\begin{aligned}
& M_{i-n_{0}} \dot{\tilde{\omega}}_{g_{i-n_{0}}} \tilde{\omega}_{g_{i-n_{0}}} \\
& \quad=P_{M_{i-n_{0}}} \dot{\theta}_{i}-\sum_{j=1}^{n} B_{i j} V_{i} V_{j} \sin \left(\theta_{i}-\theta_{j}\right) \dot{\theta}_{i} .
\end{aligned}
$$

Multiply the real power equations (10) by $\dot{\theta}_{i}$, taking account of (1)

$$
0=\sum_{j=1}^{n} B_{i j} V_{i} V_{j} \sin \left(\theta_{i}-\theta_{j}\right) \dot{\theta}_{i}+P_{d_{i}}^{0} \dot{\theta}_{i} .
$$

Multiply (5) by $\frac{1}{Q_{t_{i}}^{0}}\left(\frac{x_{q_{i}}}{T_{q_{i}}}+\frac{Q_{t_{i}}^{0} \ln \left(\frac{V_{i}}{\gamma_{i}}\right)}{T_{q_{i}}}\right)\left(=-\frac{\dot{x}_{q_{i}}}{Q_{t_{i}}^{0}}\right)$ giving

$$
\begin{aligned}
\dot{x}_{q_{i}} \ln \left(\frac{V_{i}}{\gamma_{i}}\right)+\frac{\dot{x}_{q_{i}} x_{q_{i}}}{Q_{t_{i}}^{0}}= & \frac{1}{Q_{t_{i}}^{0}}\left(-x_{q_{i}}+Q_{s_{i}}^{0}-Q_{t_{i}}\left(V_{i}\right)\right) \\
& \times\left(\frac{x_{q_{i}}}{T_{q_{i}}}+\frac{Q_{t_{i}}^{0} \ln \left(\frac{V_{i}}{\gamma_{i}}\right)}{T_{q_{i}}}\right) .
\end{aligned}
$$

Sum (23) over all the load buses. Since $\dot{V}_{i} \equiv 0$ at all generator internal buses, the summation involving $B_{i j}$ terms can be performed over all buses. Hence, using (6) we obtain

$$
\begin{aligned}
0= & \sum_{i=1}^{n_{0}-m} \frac{Q_{t_{i}}\left(V_{i}\right) \dot{V}_{i}}{V_{i}}+\sum_{i=1}^{n_{0}-m} \frac{x_{q_{i}} \dot{V}_{i}}{V_{i}}-\sum_{i=1}^{n_{0}} B_{i i} \dot{V}_{i} V_{i} \\
& -\sum_{i=1}^{n} \sum_{j=1, \neq i}^{n} B_{i j} \dot{V}_{i} V_{j} \cos \theta_{i j}
\end{aligned}
$$

i.e.,

$$
\begin{aligned}
0= & \sum_{i=1}^{n_{0}-m} \frac{Q_{t_{i}}\left(V_{i}\right) \dot{V}_{i}}{V_{i}}+\sum_{i=1}^{n_{0}-m} \frac{x_{q_{i}} \dot{V}_{i}}{V_{i}}-\sum_{i=1}^{n_{0}} B_{i i} \dot{V}_{i} V_{i} \\
& -\sum_{i=1}^{n-1} \sum_{j=i+1}^{n} B_{i j}\left(\dot{V}_{i} V_{j}+V_{i} \dot{V}_{j}\right) \cos \theta_{i j} .
\end{aligned}
$$

Sum (24) over the $m$ machine equations

$$
\begin{aligned}
& \sum_{i=1}^{m} M_{i} \dot{\tilde{\omega}}_{g_{i}} \tilde{\omega}_{g_{i}}-\sum_{i=n_{0}+1}^{n} P_{M_{i-n_{0}}} \dot{\theta}_{i} \\
& \quad+\sum_{i=n_{0}+1}^{n} \sum_{j=1}^{n} B_{i j} V_{i} V_{j} \sin \theta_{i j} \dot{\theta}_{i}=0 .
\end{aligned}
$$

Sum (25) over the $n_{0}$ real power flow equations

$$
\sum_{i=1}^{n_{0}} P_{d_{i}}^{0} \dot{\theta}_{i}+\sum_{i=1}^{n_{0}} \sum_{j=1}^{n} B_{i j} V_{i} V_{j} \sin \theta_{i j} \dot{\theta}_{i}=0 .
$$

Sum (26) over the load buses

$$
\begin{aligned}
& \sum_{i=1}^{n_{0}-m} \dot{x}_{q_{i}} \ln \left(\frac{V_{i}}{\gamma_{i}}\right)+\sum_{i=1}^{n_{0}-m} \frac{\dot{x}_{q_{i}} x_{q_{i}}}{Q_{t_{i}}^{0}} \\
& =\sum_{i=1}^{n_{0}-m} \frac{1}{Q_{t_{i}}^{0} T_{q_{i}}}\left(-x_{q_{i}}+Q_{s_{i}}^{0}-Q_{t_{i}}\left(V_{i}\right)\right) \\
& \quad \times\left(x_{q_{i}}+Q_{t_{i}}^{0} \ln \left(\frac{V_{i}}{\gamma_{i}}\right)\right)
\end{aligned}
$$


Adding (28) and (29) gives

$$
\begin{aligned}
& \sum_{i=1}^{m} M_{i} \dot{\tilde{\omega}}_{g_{i}} \tilde{\omega}_{g_{i}}-\sum_{i=n_{0}+1}^{n} P_{M_{i-n_{0}}} \dot{\theta}_{i}+\sum_{i=1}^{n_{0}} P_{d_{i}}^{0} \dot{\theta}_{i} \\
& +\sum_{i=1}^{n-1} \sum_{j=i+1}^{n} B_{i j} V_{i} V_{j} \sin \theta_{i j} \dot{\theta}_{i j}=0 .
\end{aligned}
$$

If we add this equation to (27) and (30), we obtain

$$
\begin{aligned}
\sum_{i=1}^{m} & M_{i} \dot{\tilde{\omega}}_{g_{i}} \tilde{\omega}_{g_{i}}-\sum_{i=n_{0}+1}^{n} P_{M_{i-r_{0}}} \dot{\theta}_{i}+\sum_{i=1}^{n_{0}} P_{d_{i}}^{0} \dot{\theta}_{i} \\
& +\sum_{i=1}^{n_{0}-m} \frac{Q_{t_{i}}\left(V_{i}\right) \dot{V}_{i}}{V_{i}}+\sum_{i=1}^{n_{0}-m} \frac{\dot{x}_{q_{i}} x_{q_{i}}}{Q_{t_{i}}^{0}} \\
& +\sum_{i=1}^{n_{0}-m}\left(\dot{x}_{q_{i}} \ln \left(\frac{V_{i}}{\gamma_{i}}\right)+\frac{x_{q_{i}} \dot{V}_{i}}{V_{i}}\right)-\sum_{i=1}^{n_{0}} B_{i i} \dot{V}_{i} V_{i} \\
+ & \sum_{i=1}^{n-1} \sum_{j=i+1}^{n}\left(B_{i j} V_{i} V_{j} \sin \theta_{i j} \dot{\theta}_{i j}\right. \\
& \left.=\sum_{i=1}^{n_{0}-m} \frac{1}{Q_{t_{i}}^{0} T_{q_{i}}}\left(-\dot{V}_{i} V_{j}+V_{i} \dot{V}_{j}\right) \cos \theta_{i j}\right) \\
& \times\left(x_{q_{i}}+Q_{t_{i}}^{0} \ln \left(\frac{V_{i}}{\gamma_{i}}\right)\right) .
\end{aligned}
$$

It follows via integration of the left-hand side (LHS) that

$$
\begin{aligned}
\frac{d}{d t} & {\left[\frac{1}{2} \sum_{i=1}^{m} M_{i} \tilde{\omega}_{g_{i}}^{2}-\sum_{i=n_{0}+1}^{n} P_{M_{i-n_{0}}} \theta_{i}+\sum_{i=1}^{n_{0}} P_{d_{i}}^{0} \theta_{i}\right.} \\
& -\frac{1}{2} \sum_{i=1}^{n} B_{i i} V_{i}^{2}-\sum_{i=1}^{n-1} \sum_{j=i+1}^{n} B_{i j} V_{i} V_{j} \cos \theta_{i j} \\
& \left.+\sum_{i=1}^{n_{0}-m}\left(\frac{x_{q_{i}}{ }^{2}}{2 Q_{t_{i}}^{0}}+x_{q_{i}} \ln \left(\frac{V_{i}}{\gamma_{i}}\right)+\frac{Q_{t_{i}}^{0}}{2}\left(\ln \left(\frac{V_{i}}{\mu_{i}}\right)\right)^{2}\right)\right] \\
& =-\sum_{i=1}^{n_{0}-m} \frac{T_{q_{i}}\left(\dot{x}_{q_{i}}\right)^{2}}{Q_{t_{i}}^{0}} \\
& \equiv \frac{d \mathcal{V}}{d t}
\end{aligned}
$$

The right-hand side (RHS) of (31) follows from (5) and (9).

Integrating the LHS of (31) from $t=t^{s}$ where $\underline{\tilde{\omega}}_{g}=0$, $\underline{\theta}=\underline{\theta}^{s}, \underline{V}=\underline{V}^{s}$ and $\underline{x}_{q}=\underline{x}_{q}^{s}$ results in the energy function

$$
\begin{aligned}
& \mathcal{V}\left(\underline{\tilde{\omega}}_{g}, \underline{\theta}, \underline{x}_{q}, \underline{V}, \underline{\theta}^{s}, \underline{x}_{q}^{s}, \underline{V}^{s}\right)=\frac{1}{2} \sum_{i=1}^{m} M_{i} \tilde{\omega}_{g_{i}}^{2} \\
& \quad-\sum_{i=n_{0}+1}^{n} P_{M_{i-n_{0}}}\left(\theta_{i}-\theta_{i}^{s}\right)+\sum_{i=1}^{n_{0}} P_{d_{i}}^{0}\left(\theta_{i}-\theta_{i}^{s}\right) \\
& \quad-\frac{1}{2} \sum_{i=1}^{n} B_{i i}\left(V_{i}^{2}-V_{i}^{s}\right) \\
& \quad-\sum_{i=1}^{n-1} \sum_{j=i+1}^{n} B_{i j}\left(V_{i} V_{j} \cos \theta_{i j}-V_{i}^{s} V_{j}^{s} \cos \theta_{i j}^{s}\right)
\end{aligned}
$$

$$
\begin{aligned}
& +\sum_{i=1}^{n_{0}-m}\left(\frac{x_{q_{i}}{ }^{2}-x_{q_{i}}^{s}}{2 Q_{t_{i}}^{0}}+x_{q_{i}} \ln \left(\frac{V_{i}}{\gamma_{i}}\right)-x_{q_{i}}^{s} \ln \left(\frac{V_{i}^{s}}{\gamma_{i}}\right)\right. \\
& \left.+\frac{Q_{t_{i}}^{0}}{2}\left(\left(\ln \left(\frac{V_{i}}{\mu_{i}}\right)\right)^{2}-\left(\ln \left(\frac{V_{i}^{s}}{\mu_{i}}\right)\right)^{2}\right)\right) .
\end{aligned}
$$

The last summation of (32) can be simplified by noting that $x_{q_{i}}^{s}=Q_{s_{i}}^{0}-Q_{t_{i}}\left(V_{i}^{s}\right)=-Q_{t_{i}}^{0} \ln \left(\frac{V_{i}^{s}}{\gamma_{i}}\right)$. This gives

$$
\begin{aligned}
& \frac{x_{q_{i}}{ }^{2}-x_{q_{i}}^{s}{ }^{2}}{2 Q_{t_{i}}^{0}}+x_{q_{i}} \ln \left(\frac{V_{i}}{\gamma_{i}}\right)-x_{q_{i}}^{s} \ln \left(\frac{V_{i}^{s}}{\gamma_{i}}\right) \\
& =\frac{\left(x_{q_{i}}-x_{q_{i}}^{s}\right)^{2}}{2 Q_{t_{i}}^{0}}-\frac{x_{q_{i}}^{s}}{Q_{t_{i}}^{0}}+\frac{x_{q_{i}} x_{q_{i}}^{s}}{Q_{t_{i}}^{0}}+x_{q_{i}} \ln \left(\frac{V_{i}}{\gamma_{i}}\right)+x_{q_{i}}^{s} \frac{x_{q_{i}}^{s}}{Q_{t_{i}}^{0}} \\
& =\frac{\left(x_{q_{i}}-x_{q_{i}}^{s}\right)^{2}}{2 Q_{t_{i}}^{0}}+x_{q_{i}} \ln \left(\frac{V_{i}}{V_{i}^{s}}\right) .
\end{aligned}
$$

The energy function then becomes

$$
\begin{aligned}
& \mathcal{V}\left(\underline{\tilde{\omega}}_{g}, \underline{\theta}, \underline{x}_{q}, \underline{V}, \underline{\theta}^{s}, \underline{x}_{q}^{s}, \underline{V}^{s}\right) \\
&= \frac{1}{2} \sum_{i=1}^{m} M_{i} \tilde{\omega}_{g_{i}}^{2}+\frac{1}{2} \sum_{i=1}^{n_{0}-m} \frac{\left(x_{q_{i}}-x_{q_{i}}^{s}\right)^{2}}{Q_{t_{i}}^{0}} \\
&-\sum_{i=n_{0}+1}^{n} P_{M_{i-n_{0}}}\left(\theta_{i}-\theta_{i}^{s}\right)+\sum_{i=1}^{n_{0}} P_{d_{i}}^{0}\left(\theta_{i}-\theta_{i}^{s}\right) \\
&-\frac{1}{2} \sum_{i=1}^{n} B_{i i}\left(V_{i}^{2}-V_{i}^{s}\right) \\
&-\sum_{i=1}^{n-1} \sum_{j=i+1}^{n} B_{i j}\left(V_{i} V_{j} \cos \theta_{i j}-V_{i}^{s} V_{j}^{s} \cos \theta_{i j}^{s}\right) \\
&+\sum_{i=1}^{n_{0}-m}\left(x_{q_{i}} \ln \left(\frac{V_{i}}{V_{i}^{s}}\right)^{2}\right. \\
&+\frac{Q_{t_{i}}^{0}}{2}\left(\left(\ln \left(\frac{V_{i}}{\mu_{i}}\right)^{2}-\left(\ln \left(\frac{V_{i}^{s}}{\mu_{i}}\right)\right)^{2}\right)\right) .
\end{aligned}
$$

From (31) it can be seen that $\dot{\mathcal{V}}$ is negative semidefinite. Assume that $\dot{\mathcal{V}} \equiv 0$ for all $t$. Then from (31), $\dot{x}_{q_{i}}=0$ for $i=1, \cdots, n_{0}-m$, i.e., for all buses at which there are dynamic loads. Equations (5) and (6) therefore imply that voltage magnitudes and reactive power loads at those buses are constant. By definition, reactive power loads at buses $i=n_{0}-m+1, \cdots, n_{0}$ are constant. Also, real power loads at all load buses are constant. Therefore when $\dot{\mathcal{V}} \equiv 0$, solutions satisfy $2 n_{0}$ steady-state power balance equations. Generically, because $V_{i}, i=1, \cdots, n_{0}-m$ are constant, then $\underline{\theta}_{g}$ must also be constant, so the system is in steady-state. Therefore there exists no nontrivial trajectory over which $\dot{\mathcal{V}} \equiv$ 0 . This means that the function $\mathcal{V}$ can be used to establish the stability properties of an equilibrium point. If for example $\mathcal{V}$ is positive definite about the equilibrium point then that point is asymptotically stable. Conditions for positive definiteness are examined in Section IV-C.

It should be remembered that $\mathcal{V}$ can only be a Lyapunov function if $Q_{t_{i}}\left(V_{i}\right)$ has the form given by (7). This is because of the mathematical constraints involved in formulating $\mathcal{V}$. It was necessary to obtain a path-independent integral on the 
LHS of (31), whilst maintaining a nonpositive expression on the RHS.

\section{POPOV CRITERION ANALYSIS OF THE COA MODEL}

\section{A. Stability Criterion Background}

The development of Lyapunov functions using the Popov stability criterion is based upon establishing the system equations in the form

$$
\underline{\dot{x}}=F \underline{x}-G \underline{\psi}\left(H^{t} \underline{x}\right) .
$$

The transfer function of the linear part of the system is

$$
G_{L}(s)=H^{t}(s I-F)^{-1} G \text {. }
$$

Consider the modified transfer function

$$
G_{M}(s)=(N+Q s) G_{L}(s)
$$

where $(N+Q s)$ does not give any pole-zero cancellations with $G_{L}(s)$, and $N \geq 0$ and $Q>0$. Based on results of [1], [12], and [13], if $G_{M}(s)$ is positive real then there exist real matrices $P, L$, and $W$ with $P$ positive definite symmetric, such that

$$
\begin{aligned}
P F+F^{t} P & =-L L^{t} \\
P G & =H N+F^{t} H Q-L W \\
W^{t} W & =Q H^{t} G+G^{t} H Q .
\end{aligned}
$$

The Lyapunov function subsequently generated is

$$
\begin{aligned}
\mathcal{V}\left(\underline{x}-\underline{x}^{s}\right)= & \frac{1}{2}\left(\underline{x}-\underline{x}^{s}\right)^{t} P\left(\underline{x}-\underline{x}^{s}\right) \\
& +\int_{H^{t} \underline{x}^{s}}^{H^{t} \underline{x}}\left[\underline{\psi}(\underline{\xi})-\underline{\psi}\left(H^{t} \underline{x}^{s}\right)\right]^{t} Q d \underline{\xi}
\end{aligned}
$$

The derivative of this function along system trajectories is

$$
\begin{aligned}
\dot{\mathcal{V}}(\underline{y})= & -\frac{1}{2}\left(L^{t} \underline{y}-W \underline{\phi}\left(H^{t} \underline{y}\right)\right)^{t}\left(L^{t} \underline{y}-W \underline{\phi}\left(H^{t} \underline{y}\right)\right) \\
& -\underline{y}^{t} H N \underline{\phi}\left(H^{t} \underline{y}\right)
\end{aligned}
$$

where $\underline{y}=\underline{x}-\underline{x}^{s}$ and $\underline{\phi}\left(H^{t} \underline{y}\right)=\underline{\psi}\left(H^{t}\left(\underline{y}+\underline{x}^{s}\right)\right)-\underline{\psi}\left(H^{t} \underline{x}^{s}\right)$.

\section{B. Lyapunov Function Development}

In order to match the system equations (17)-(20) to the form (34) we define

$$
\begin{aligned}
\underline{x} & =\left[\underline{\underline{\omega}}_{g}^{t} \underline{\theta}^{t} \underline{x}_{q}^{t} \underline{V}^{t}\right]^{t} \\
\underline{\psi}\left(H^{t} \underline{x}\right) & =\left[\begin{array}{c}
\underline{\theta}, \underline{V})-\underline{P}^{0} \\
\underline{k}(\underline{V}) \\
{[V]^{-1}\left(\underline{Q} \underline{\underline{Q}}(\underline{\theta}, \underline{V})+\underline{Q}_{d}\left(\underline{x}_{q}, \underline{V}\right)\right)}
\end{array}\right] \\
& =\left[\begin{array}{c}
\underline{f}(\underline{\theta}, \underline{V}) \\
\underline{k}(\underline{V}) \\
g\left(\underline{\theta}, \underline{x}_{q}, \underline{V}\right)
\end{array}\right] \\
H^{t} & =\left[\begin{array}{cccc}
0 & I_{n} & 0 & 0 \\
0 & 0 & I_{n_{0}-m} & 0 \\
0 & 0 & 0 & I_{n_{0}}
\end{array}\right]
\end{aligned}
$$

$$
\begin{aligned}
F & =\left[\begin{array}{cccc}
0 & 0 & 0 & 0 \\
R_{2} & 0 & 0 & 0 \\
0 & 0 & -T_{q}^{-1} & 0 \\
0 & 0 & 0 & 0
\end{array}\right] \\
G & =\left[\begin{array}{ccc}
M_{g}^{-1} R_{2}^{t} & 0 & 0 \\
R_{1} D_{l}^{-1} R_{1}^{t} & 0 & 0 \\
0 & T_{q}^{-1} Q_{t}^{0} & 0 \\
0 & 0 & E^{-1}
\end{array}\right] .
\end{aligned}
$$

The transfer function $G_{L}(s)$ then has a block diagonal structure, with the diagonal blocks given by

$$
\begin{aligned}
& G_{L}(s)_{11}=\frac{1}{s}\left(\frac{1}{s} R_{2} M_{g}^{-1} R_{2}^{t}+R_{1} D^{-1} R_{1}^{t}\right) \\
& G_{L}(s)_{22}=\left(s I_{n_{0}-m}+T_{q}^{-1}\right)^{-1} T_{q}^{-1} Q_{t}^{0} \\
& G_{L}(s)_{33}=\frac{1}{s} E^{-1} .
\end{aligned}
$$

We require the transfer function $G_{M}(s)$ to be positive real. Let

$$
\begin{aligned}
N & =\left[\begin{array}{ccc}
n_{1} I_{n} & 0 & 0 \\
0 & n_{3} I_{n_{0}-m} & 0 \\
0 & 0 & n_{2} I_{n_{0}}
\end{array}\right] \\
Q & =\left[\begin{array}{ccc}
q_{1} I_{n} & 0 & 0 \\
0 & q_{3} I_{n_{0}-m} & 0 \\
0 & 0 & q_{2} I_{n_{0}}
\end{array}\right] .
\end{aligned}
$$

Conditions were established in [13] which ensured $G_{M}(s)$ was positive real for the static load case. Those conditions were $n_{1}=0$ and $q_{1} \geq 0$. In addition to these conditions we require that the transfer function

$$
Z(s)=\left(n_{3}+q_{3} s\right) I_{n_{0}-m}\left(s I_{n_{0}-m}+T_{q}^{-1}\right)^{-1} T_{q}^{-1} Q_{t}^{0}
$$

be positive real. This is satisfied if the transfer functions

$$
Z_{i}(s)=\frac{\left(n_{3}+q_{3} s\right) Q_{t_{i}}^{0}}{T_{q_{i}} s+1}
$$

are positive real. It can be shown that

$$
\operatorname{Re}\left\{Z_{i}(j \omega)\right\}=\frac{Q_{t_{i}}^{0}\left(n_{3}+q_{3} T_{q_{i}} \omega^{2}\right)}{\left(T_{q_{i}} \omega\right)^{2}+1}
$$

Hence, $Z(s)$ is positive real for any $n_{3} \geq 0$ and $q_{3} \geq 0$. It will be shown in Section IV-D that it is necessary also to take $q_{1}=q_{2}=q_{3}=q$.

If $(F, G, H)$ was a minimal state-space representation of $G_{L}(s)$, the Popov criterion would imply the existence of matrices $P, L$ and $W$ which satisfy (37)-(39). For the COA model, minimality is not satisfied, so we assume their existence. 
The rigorous development of Lyapunov functions for the COA model is documented in [6]. That analysis leads to

$$
\begin{aligned}
P & =\left[\begin{array}{cccc}
q M_{g} & 0 & 0 & 0 \\
0 & 0 & 0 & 0 \\
0 & 0 & q\left(Q_{t}^{0}\right)^{-1} & 0 \\
0 & 0 & 0 & n_{2} E
\end{array}\right] \\
L & =\left[\begin{array}{cccc}
0 & 0 & 0 & 0 \\
0 & 0 & 0 & 0 \\
0 & -\sqrt{2 q} T_{q}^{-\frac{1}{2}}\left(Q_{t}^{0}\right)^{-\frac{1}{2}} & 0 & 0 \\
0 & 0 & 0 & 0
\end{array}\right] \\
W & =\left[\begin{array}{ccc}
\sqrt{2 q} D_{l}^{-\frac{1}{2}} R_{1}^{t} & 0 & 0 \\
0 & \sqrt{2 q} T_{q}^{-\frac{1}{2}}\left(Q_{t}^{0}\right)^{\frac{1}{2}} & 0 \\
0 & 0 & 0 \\
0 & 0 & \sqrt{2 q} E^{-\frac{1}{2}}
\end{array}\right]
\end{aligned}
$$

In obtaining $P, L$ and $W$, it was convenient to let $n_{3}=0$. An algebraic Riccati equation was solved to find the unique solution for $P_{33}$.

The Lyapunov function candidate developed from the Popov criterion analysis is therefore given by

$$
\begin{aligned}
\mathcal{V}\left(\underline{x}-\underline{x}^{s}\right) & =\frac{1}{2}\left(\underline{x}-\underline{x}^{s}\right)^{t}\left[\begin{array}{cccc}
q M_{g} & 0 & 0 & 0 \\
0 & 0 & 0 & 0 \\
0 & 0 & q\left(Q_{t}^{0}\right)^{-1} & 0 \\
0 & 0 & 0 & n_{2} E
\end{array}\right]\left(\underline{x}-\underline{x}^{s}\right) \\
& +q \int_{H^{t} \underline{x}^{s}}^{H^{t} \underline{x}}[\underline{\psi} \underline{\xi})-\underline{\left.\psi\left(H^{t} \underline{x}^{s}\right)\right]^{t} d \underline{\xi}}
\end{aligned}
$$

In the system model (17)-(20), the singular perturbations $D_{l_{i}} \dot{\theta}_{i}$ and $\epsilon_{i} \dot{V}_{i}$ were introduced on the power balance equations so that the model took on the differential equation form required for the Popov criterion analysis. Having obtained the desired Lyapunov function candidate, we shall now allow $D_{l} \rightarrow 0$ and $E \rightarrow 0$ in (17)-(20) and in (41). Also, with $E \rightarrow 0$ it can be seen from (41) that $q$ acts as a scaling factor. Without loss of generality, we can therefore let $q=1$. The resulting function

$$
\begin{aligned}
\mathcal{V}\left(\underline{x}-\underline{x}^{s}\right)= & \frac{1}{2} \tilde{\omega}_{g}^{t} M_{g} \underline{\tilde{\omega}}_{g}+\frac{1}{2}\left(\underline{x}_{q}-\underline{x}_{q}^{s}\right)^{t}\left(Q_{t}^{0}\right)^{-1}\left(\underline{x}_{q}-\underline{x}_{q}^{s}\right) \\
& +\int_{H^{t} \underline{x}^{s}}^{H^{t} \underline{x}}\left[\underline{\psi}(\underline{\xi})-\underline{\psi}\left(H^{t} \underline{x}^{s}\right)\right]^{t} d \underline{\xi}
\end{aligned}
$$

can be interpreted as a Lyapunov function candidate for the corresponding differential-algebraic system [16], [17].

\section{Validity of the Lyapunov Function}

The precise conditions required for the Popov stability criterion have not been met exactly. There may be polezero cancellation between $(N+Q s)$ and $G_{L}(s)$, and $\underline{\psi}($. is not (globally) strictly passive. Also, the COA model is not minimal. Therefore it is important to check that the function (42) satisfies Lyapunov function properties. The first property relates to the (local) positive definiteness of the function.
Lemma 1: The function $\mathcal{V}\left(\underline{x}-\underline{x}^{s}\right)$ given by (42) is locally positive definite in a neighborhood of the equilibrium $\underline{x}^{s}$ of the system (17)-(20) if the matrix

$$
\begin{aligned}
\mathcal{H} & =\left[\begin{array}{cccc}
M_{g} & 0 & 0 & 0 \\
0 & \frac{\partial \underline{f}(\underline{\theta}, \underline{V})}{\partial \underline{\theta}} & 0 & \frac{\partial \underline{f}(\underline{\theta}, \underline{V})}{\partial \underline{V}} \\
0 & 0 & \left(Q_{t}^{0}\right)^{-1} & \frac{\partial \underline{\underline{k}}(\underline{\underline{V}})}{\partial \underline{V}} \\
0 & \frac{\partial \underline{g}\left(\underline{\theta}, \underline{x}_{q}, \underline{V}\right)}{\partial \underline{\theta}} & \frac{\partial \underline{g}\left(\underline{\theta}, \underline{x}_{q}, \underline{V}\right)}{\partial \underline{x}_{q}} & \frac{\partial \underline{g}\left(\underline{\underline{\theta}}, \underline{x}_{q}, \underline{V}\right)}{\partial \underline{V}}
\end{array}\right] \\
& =\left[\begin{array}{cccc}
M_{g} & 0 & 0 & 0 \\
0 & \underline{f}_{\theta} & 0 & \underline{f}_{v} \\
0 & 0 & \left(Q_{t}^{0}\right)^{-1} & \underline{\underline{k}}_{v} \\
0 & \underline{g}_{\theta} & \underline{g}_{x_{q}} & \underline{g}_{v}
\end{array}\right]
\end{aligned}
$$

evaluated at the equilibrium point $\underline{x}^{s}$ is positive definite.

The proof of this lemma follows immediately from similar results in [7] and [17].

Notice that $\mathcal{H}$ is positive definite iff

$$
J=\left.\left[\begin{array}{ccc}
\underline{f}_{\theta} & 0 & \underline{f}_{v} \\
0 & \left(Q_{t}^{0}\right)^{-1} & \underline{k}_{v} \\
\underline{g}_{\theta} & \underline{g}_{x_{q}} & \underline{g}_{v}
\end{array}\right]\right|_{\underline{x}=\underline{x}^{s}}
$$

is positive definite. But $J$ is the Jacobian of the equations describing equilibria of the system (17)-(20). Further, because the principal diagonal submatrices of a positive definite matrix are themselves positive definite, it follows by rearranging rows and columns that $J$ can only be positive definite if $\left.\underline{g}_{v}\right|_{\underline{x}}=\underline{x}^{s}$ is positive definite, and if

$$
J_{t}=\left.\left[\begin{array}{ll}
\underline{f}_{\theta} & \underline{f}_{v} \\
\underline{g}_{\theta} & \underline{g}_{v}
\end{array}\right]\right|_{\underline{x}=\underline{x}^{s}}
$$

is positive definite. Matrix $J_{t}$ is the Jacobian of (scaled) power flow equations, in which the reactive power demand is effectively given by the transient characteristic $\underline{Q}_{t}(\underline{V})$ [18], [23], i.e.,

$$
\begin{aligned}
\underline{g}_{v} & =\frac{\partial}{\partial V}\left([V]^{-1}\left(\underline{Q}_{l}(\underline{\theta}, \underline{V})+\underline{x}_{q}+\underline{Q}_{t}(\underline{V})\right)\right) \\
& =[V]^{-1} \frac{\partial}{\partial V}\left(\underline{Q}_{l}(\underline{\theta}, \underline{V})+\underline{Q}_{t}(\underline{V})\right)
\end{aligned}
$$

at an equilibrium point. Hence the subscript $t$.

The following result also establishes a connection between the positive definiteness of $J$ and the "steady-state" Jacobian $J_{s}$.

Theorem 2: $J$ is positive definite iff the Jacobian

$$
J_{s}=\left.\left[\begin{array}{ll}
\underline{f}_{\theta} & \underline{f}_{v} \\
\underline{g}_{\theta} & \underline{g}_{v}^{*}
\end{array}\right]\right|_{\underline{x}_{=x^{s}}}
$$

is positive definite, where $\underline{g}^{*}(\underline{\theta}, \underline{V})=[V]^{-1}\left(\underline{Q}_{l}(\underline{\theta}, \underline{V})+\underline{Q}_{s}^{0}\right)$.

The proof of this theorem is given in Appendix I.

The matrix $J_{s}$ is the Jacobian of the power flow equations in which reactive power demand is given by the steady-state values $\underline{Q}_{s}^{0}$, i.e., the normal power flow Jacobian. Hence we can conclude that at equilibrium points where $J_{s}$ is positive definite, $J$ and $J_{t}$ will also be positive definite, and the Lyapunov function (42) will be (locally) positive definite. This condition on $J_{s}$ corresponds to local regularity of the 
equilibrium point [18], and is generally satisfied at normal operating points. Further, the positive definiteness of $J_{s}$ relates to conditions established in [18] for small disturbance stability of load systems.

The second condition which must be checked to ensure the validity of the Lyapunov function is that the derivative of the Lyapunov function along trajectories is locally nonpositive about the operating point. This derivative is given by

$$
\begin{aligned}
\dot{\mathcal{V}}(\underline{y})= & -\frac{1}{2}\left(L^{t} \underline{y}-W \underline{\phi}\left(H^{t} \underline{y}\right)\right)^{t}\left(L^{t} \underline{y}-W \underline{\phi}\left(H^{t} \underline{y}\right)\right) \\
& -\underline{y}^{t} H N \underline{\phi}\left(H^{t} \underline{y}\right)
\end{aligned}
$$

where $y=\underline{x}-\underline{x}^{s}$ and $\phi\left(H^{t} y\right)=\psi\left(H^{t}\left(\underline{y}+\underline{x}^{s}\right)\right)-\psi\left(H^{t} \underline{x}^{s}\right)$. With $n_{1}=0$, the last term in this equation evaluates to

$$
-n_{2}\left(\underline{V}-\underline{V}^{s}\right)^{t} \underline{g}\left(\underline{\theta}, \underline{x}_{q}, \underline{V}\right)-n_{3}\left(\underline{x}_{q}-\underline{x}_{q}^{s}\right)^{t}\left(\underline{k}(\underline{V})-\underline{k}\left(\underline{V}^{s}\right)\right) \text {. }
$$

In general, to ensure this term remains nonpositive we require $n_{2}=n_{3}=0$. In the development of the Lyapunov function candidate, we arbitrarily set $n_{3}=0$. We have now established that that condition is mandatory.

Consider trajectories along which $\dot{\mathcal{V}}(\underline{y}) \equiv 0$. Then from (45)

$$
\begin{aligned}
& L^{t} \underline{\underline{y}}-W \underline{\phi}\left(H^{t} \underline{y}\right) \\
& \left.\quad=\left[\begin{array}{c}
L_{32}^{t}\left(\underline{x}_{q}-\underline{x}_{q 1}^{s}\right)-W_{22}(\underline{f}(\cdot) \\
0 \\
-W_{43} \underline{g}(\cdot)
\end{array}\right]=0 . \underline{k}\left(\underline{V}^{s}\right)\right)
\end{aligned}
$$

Consider initially the singularly perturbed system, with $D_{l} \neq$ $0, E \neq 0$. Then (46) implies

$$
\begin{aligned}
\underline{f}_{l}(\underline{\theta}, \underline{V}) & =0 \\
\underline{g}\left(\underline{\theta}, \underline{x}_{q}, \underline{V}\right) & =0 \\
L_{32}^{t}\left(\underline{x}_{q}-\underline{x}_{q}^{s}\right) & =W_{22}\left(\underline{k}(\underline{V})-k\left(\underline{V}^{s}\right)\right) .
\end{aligned}
$$

Substituting $L_{32}$ and $W_{22}$ into (49) gives

$$
\begin{aligned}
\underline{x}_{q}-\underline{x}_{q}^{s} & =-Q_{t}^{0}\left(\underline{k}(\underline{V})-\underline{k}\left(\underline{V}^{s}\right)\right) \\
& =T_{q} \underline{\dot{x}}_{q}+\underline{x}_{q}-\underline{x}_{q}^{s} .
\end{aligned}
$$

So $\underline{\dot{x}}_{q}=0$, and hence $\underline{x}_{q}=\underline{x}_{q}^{s}$. Substituting (47) and (48) into the system model equations (18), (20) results in $\dot{V}=0$ and $\underline{\dot{\theta}}_{l}=0$. This implies $\underline{V}=\underline{V}^{s}$ and $\underline{\theta}_{l}=\underline{\theta}_{l}^{s}$. It follows from an argument similar to that presented at the end of Section III that $\underline{\theta}=\underline{\theta}^{s}$. Hence $\underline{\dot{\theta}}_{g}=\underline{\tilde{\omega}}_{g}=0$.

In the differential-algebraic (DA) system which results when $D_{l} \rightarrow 0$ and $E \rightarrow 0$, (47) and (48) are satisfied explicitly. Equation (49) again follows from (46), so $\underline{\dot{x}}_{q}=0$, and $\underline{x}_{q}=\underline{x}_{q}^{s}, V_{i}=V_{i}^{s}, i=1, \cdots, n_{0}-m$. In this case we cannot state directly that $\underline{V}=\underline{V}^{s}$ and $\underline{\theta}_{l}=\underline{\theta}_{l}^{s}$. However the same argument as presented at the end of Section III allows us to conclude that generically $\dot{\mathcal{V}}(\underline{y}) \equiv 0$ only at isolated equilibria. The positive-definiteness of the DA energy function is inherited from the energy function for the singularly perturbed system, since the function is the same except for the additional constraints (47) and (48).

In both the DA and singularly perturbed cases, under certain generic conditions we can conclude that $\dot{\mathcal{V}}(\underline{y}) \equiv 0$ implies $\underline{x}=\left[\underline{\tilde{\omega}}_{g}^{t} \underline{\theta}^{t} \underline{x}_{q}^{t} \underline{V}^{t}\right]^{t}=\left[\underline{0}^{t} \underline{\theta}^{s t} \underline{x}_{q}^{s t} \underline{V}^{s t}\right]^{t}=\underline{x}^{s}$. Therefore if
$J_{s}$ is positive definite, it follows from LaSalle's Invariance Theorem [33] that $\underline{x}^{s}$ is asymptotically stable. The formal proof is similar to a result presented in [13]. (Recall that the singularly perturbed system with COA coordinates does not have a physical interpretation. However the energy function for the DA system may be used for stability assessment.)

The set of Lyapunov functions (41) generated in Section IV$\mathrm{B}$ was derived under the assumption $n_{3}=0$. However, as we saw above, that condition was necessary to ensure $\dot{\mathcal{V}} \leq$ 0 . Further, the free parameter $q$ was only a scalar multiplier which could be factored from the functions. Therefore we conclude that there is only one Lyapunov function which may be generated in this way for the COA model. The damping provided by the dynamic loads does not appear to give any extra freedom in forming a Lyapunov function. (This is in contrast with the case of machine damping where the damping introduces some freedom in the Lyapunov function. Section VB considers this case.)

\section{Conditions for Integral Path-Independence}

The Lyapunov function integral term

$$
W=\int_{H^{t} \underline{x}^{s}}^{H^{t} \underline{x}}\left[\underline{\psi}(\underline{\xi})-\underline{\psi}\left(H^{t} \underline{x}^{s}\right)\right]^{t} Q d \underline{\xi}
$$

is path-independent iff the following conditions hold:

$$
\frac{\partial \underline{f}}{\partial \underline{\theta}}, \frac{\partial \underline{k}}{\partial \underline{x}_{q}} \text { and } \frac{\partial \underline{g}}{\partial \underline{V}} \text { are symmetric }
$$

and

$$
\begin{aligned}
& q_{1} \frac{\partial \underline{f}}{\partial \underline{V}}=q_{2}\left[\frac{\partial \underline{g}}{\partial \underline{\theta}}\right]^{t} \\
& q_{1} \frac{\partial \underline{f}}{\partial \underline{x}_{q}}=q_{3}\left[\frac{\partial \underline{k}}{\partial \underline{\theta}}\right]^{t} \\
& q_{3} \frac{\partial \underline{k}}{\partial \underline{V}}=q_{2}\left[\frac{\partial \underline{g}}{\partial \underline{x}_{q}}\right]^{t} .
\end{aligned}
$$

Due to the structure of $\underline{f}, \underline{g}$ and $\underline{k}$, all of these conditions are satisfied if $q_{1}=q_{2}=q_{3}=q$. Hence our earlier requirement that $q_{1}=q_{2}=q_{3}=q$.

\section{E. Evaluating the Lyapunov Function}

The desired Lyapunov function (40) is composed of a quadratic term and an integral term. The quadratic term was given in (42). The integral term

$$
\begin{aligned}
W & \left(\underline{\theta}, \underline{x}_{q}, \underline{V}, \underline{\theta}^{s}, \underline{x}_{q}^{s}, \underline{V}^{s}\right) \\
\quad & =\int_{\left(\underline{\theta}^{s}, \underline{x}_{q}^{s}, \underline{V}^{s}\right)}^{\left(\underline{\theta}, \underline{x}_{q}, \underline{V}\right)}\left[\underline{\psi}(\underline{\xi})-\underline{\psi}\left(H^{t} \underline{x}^{s}\right)\right]^{t} d \underline{\xi}
\end{aligned}
$$

shall now be evaluated. It was shown in Section IV-D that this integral is path-independent. We shall therefore evaluate it by integrating with respect to one variable at a time, whilst keeping all other variables constant. Therefore

$$
\begin{aligned}
W= & \int_{\underline{\theta}^{s}}^{\underline{\theta}}\left[\underline{P}_{b}\left(\underline{\zeta}, \underline{V}^{s}\right)-\underline{P}^{0}\right]^{t} d \underline{\zeta}+\int_{\underline{x}_{q}^{s}}^{\underline{x}_{q}}\left[\underline{k}\left(\underline{V}^{s}\right)-\underline{k}\left(\underline{V}^{s}\right)\right]^{t} d \underline{s} \\
& +\int_{\underline{\underline{V}}^{s}}^{\underline{V}}\left[[z]^{-1}\left(\underline{Q}_{l}(\underline{\theta}, \underline{z})+\underline{Q}_{d}\left(\underline{x}_{q}, \underline{z}\right)\right)\right]^{t} d \underline{z} .
\end{aligned}
$$


The first integral of (50) was evaluated in [13] and [14] as $-\frac{1}{2} \sum_{i=1}^{n} \sum_{j=1}^{n} V_{i}^{s} V_{j}^{s} B_{i j}\left(\cos \theta_{i j}-\cos \theta_{i j}^{s}\right)-\sum_{i=1}^{n} P_{i}^{0}\left(\theta_{i}-\theta_{i}^{s}\right)$.

The second integral is zero. The third integral can be written

$$
\begin{aligned}
& \sum_{i=1}^{n_{0}} \int_{V_{i}^{s}}^{V_{i}} \frac{1}{z_{i}}\left(Q_{b_{i}}\left(\underline{\theta}, V_{1}^{s}, \cdots, z_{i}, \cdots, V_{n}\right)\right) d z_{i} \\
& \quad+\sum_{i=1}^{n_{0}-m} \int_{V_{i}^{s}}^{V_{i}} \frac{1}{z_{i}}\left(x_{q_{i}}+Q_{t_{i}}^{0} \ln \left(\frac{z_{i}}{\mu_{i}}\right)\right) d z_{i} .
\end{aligned}
$$

The first term of (51) was evaluated in [13] and [14]. The expression (51) becomes

$$
\begin{aligned}
& \frac{1}{2} \sum_{i=1}^{n} \sum_{j=1}^{n}\left(V_{i}^{s} V_{j}^{s}-V_{i} V_{j}\right) B_{i j} \cos \theta_{i j}+\sum_{i=1}^{n_{0}-m} x_{q_{i}} \ln \left(\frac{V_{i}}{V_{i}^{s}}\right) \\
& \quad+\sum_{i=1}^{n_{0}-m} \frac{Q_{t_{i}}^{0}}{2}\left(\left(\ln \left(\frac{V_{i}}{\mu_{i}}\right)\right)^{2}-\left(\ln \left(\frac{V_{i}^{s}}{\mu_{i}}\right)\right)^{2}\right) .
\end{aligned}
$$

When these integral terms are added with the quadratic terms of (42), we obtain the Lyapunov function

$$
\begin{aligned}
& \mathcal{V}\left(\underline{\tilde{\omega}}_{g}, \underline{\theta}, \underline{x}_{q}, \underline{V}, \underline{\theta}^{s}, \underline{x}_{q}^{s}, \underline{V}^{s}\right) \\
&= \frac{1}{2} \underline{\tilde{\omega}}_{g}^{t} M_{g} \tilde{\tilde{\omega}}_{g}+\frac{1}{2}\left(\underline{x}_{q}-\underline{x}_{q}^{s}\right)^{t}\left(Q_{t}^{0}\right)^{-1}\left(\underline{x}_{q}-\underline{x}_{q}^{s}\right) \\
&-\frac{1}{2} \sum_{i=1}^{n} \sum_{j=1}^{n} B_{i j}\left(V_{i} V_{j} \cos \theta_{i j}-V_{i}^{s} V_{j}^{s} \cos \theta_{i j}^{s}\right) \\
&-\sum_{i=1}^{n} P_{i}^{0}\left(\theta_{i}-\theta_{i}^{s}\right)+\sum_{i=1}^{n_{0}-m} x_{q_{i}} \ln \left(\frac{V_{i}}{V_{i}^{s}}\right) \\
&+\sum_{i=1}^{n_{0}-m} \frac{Q_{t_{i}}^{0}}{2}\left(\left(\ln \left(\frac{V_{i}}{\mu_{i}}\right)\right)^{2}-\left(\ln \left(\frac{V_{i}^{s}}{\mu_{i}}\right)\right)^{2}\right) .
\end{aligned}
$$

This Lyapunov function is exactly the same as the function (33) produced from first integral analysis. (The summations in (33) and (52) are expressed slightly differently though.)

It follows that $\dot{\mathcal{V}}$ given by (45) must be the same as for the first-integral analysis (31). Consider (45) and (46), recalling that $N=0$. With $q=1$, matrices $W_{11}, W_{22}$ and $L_{32}$ were evaluated as

$$
\begin{aligned}
& W_{11}=\sqrt{2} D_{l}^{-\frac{1}{2}} R_{1}^{t} \\
& W_{22}=\sqrt{2} T_{q}^{-\frac{1}{2}}\left(Q_{t}^{0}\right)^{\frac{1}{2}} \\
& L_{32}=-\sqrt{2} T_{q}^{-\frac{1}{2}}\left(Q_{t}^{0}\right)^{-\frac{1}{2}} .
\end{aligned}
$$

For the DA system, functions $\underline{f}_{l}(\cdot)$ and $g(\cdot)$ are identically zero. So by making use of the load model (19) and substituting into (45) it follows that

$$
\dot{\mathcal{V}}=-\underline{\dot{x}}_{q}^{t} T_{q}\left(Q_{t}^{0}\right)^{-1} \underline{x}_{q}
$$

which is precisely the desired result.

\section{POPOV CRITERION ANALYSIS OF THE MACHINE REFERENCE MODEL}

The COA model studied previously is convenient for the purposes of analysis. However the machine angle (MA) reference model is in a sense a more natural representation of the power system. In the MA model, all angles are referred to the angle of the $m$ th machine, i.e., the $n$th bus, rather than to a fictitious center of angle. Another benefit of the MA model over the COA model is that it allows for generator and/or load damping to be nonzero. The MA model tends to be more commonly used.

In [5], [13], [14], a Popov criterion analysis was undertaken for the machine reference model with static loads. It was found that if generator damping is present, there is some freedom in the choice of Lyapunov function. A similar analysis will be undertaken in this section for the case where reactive power loads exhibit dynamic behavior.

\section{A. Machine Reference Model}

In the machine reference model, all angles are referenced to the $n$th bus angle, i.e., $\alpha_{i}=\delta_{i}-\delta_{n}$. We therefore define

$$
T=\left[\begin{array}{ll}
I_{n-1} & -\underline{1}_{(n-1) \times 1}
\end{array}\right]=\left[\begin{array}{ll}
T_{1} & T_{2}
\end{array}\right]
$$

where $\underline{1}_{p q}$ refers to a $p \times q$ matrix with all elements equal to 1 . (In cases where the dimensions are unambiguous, the subscripts will be dropped.) Matrix $T$ is partitioned so that $T_{1}$ is an $(n-1) \times n_{0}$ matrix. We then define

$$
\underline{\alpha}=\left[\alpha_{1}, \cdots, \alpha_{n-1}\right]^{t}=T \underline{\delta}
$$

so that

$$
\underline{\dot{\alpha}}=T \underline{\dot{\delta}}=T \underline{\omega} .
$$

Also define $P_{b_{i}}(\underline{\alpha}, \underline{V}), Q_{b_{i}}(\underline{\alpha}, \underline{V})$ as the MA forms of (1), (2), respectively, and $\underline{f}(\underline{\alpha}, \underline{V}), \underline{g}\left(\underline{\alpha}, \underline{x}_{q}, \underline{V}\right)$ as the MA forms of (21), (22), respectively. Let

$$
\underline{\tilde{f}}=\left[f_{1}, \cdots, f_{n-1}\right]^{t} .
$$

The singularly perturbed form of the MA structure preserving model can then be written

$$
\begin{aligned}
\underline{\dot{\omega}}_{g} & =-M_{g}^{-1} D_{g} \underline{\omega}_{g}-M_{g}^{-1} T_{2}^{t} \underline{\tilde{f}}(\underline{\alpha}, \underline{V}) \\
\underline{\dot{\alpha}} & =T_{2} \underline{\omega}_{g}-T_{1} D_{l}^{-1} T_{1}^{t} \underline{\tilde{f}}(\underline{\alpha}, \underline{V}) \\
\underline{\dot{x}}_{q} & =-T_{q}^{-1} \underline{x}_{q}-T_{q}^{-1} Q_{t}^{0} \underline{k}(\underline{V}) \\
\dot{V} & =-E^{-1}[V]^{-1}\left(\underline{Q}_{b}(\underline{\alpha}, \underline{V})+\underline{Q}_{d}\left(\underline{x}_{q}, \underline{V}\right)\right) .
\end{aligned}
$$

Complete details of the model development can be found in a number of references, including [14], [20]. The equations are again singularly perturbed through the introduction of the term $D_{l_{i}} \dot{\alpha}_{i}$ into the real power balance equations for load buses, and the term $\epsilon_{i} \dot{V}_{i}$ into the reactive power balance equations for load buses. They are again required for the Popov criterion analysis, though may be set to zero in the final Lyapunov function. Note that in this model the $D_{l_{i}} \dot{\alpha}_{i}$ term can be physically interpreted as load damping.

\section{B. Lyapunov Function Development}

To facilitate the development of Lyapunov functions, using the Popov analysis of Section IV-A, the system equations are formulated as

$$
\underline{\dot{x}}=F \underline{x}-G \underline{\psi}\left(H^{t} \underline{x}\right)
$$


where

$$
\begin{aligned}
\underline{x} & =\left[\underline{\omega}_{g}^{t} \underline{\alpha}^{t} \underline{x}_{q}^{t} \underline{V}^{t}\right]^{t} \\
\underline{\psi}\left(H^{t} \underline{x}\right) & =\left[\begin{array}{c}
\tilde{f}(\underline{\alpha}, \underline{V}) \\
\underline{k}(\underline{V}) \\
g\left(\underline{\alpha}, \underline{x}_{q}, \underline{V}\right)
\end{array}\right] \\
H^{t} & =\left[\begin{array}{cccc}
0 & I_{n-1} & 0 & 0 \\
0 & 0 & I_{n_{0}-m} & 0 \\
0 & 0 & 0 & I_{n_{0}}
\end{array}\right] \\
F & =\left[\begin{array}{cccc}
-M_{g}^{-1} D_{g} & 0 & 0 & 0 \\
T_{2} & 0 & 0 & 0 \\
0 & 0 & -T_{q}^{-1} & 0 \\
0 & 0 & 0 & 0
\end{array}\right] \\
G & =\left[\begin{array}{cccc}
M_{g}^{-1} T_{2}^{t} & 0 & 0 \\
T_{1} D_{l}^{-1} T_{1}^{t} & 0 & 0 \\
0 & T_{q}^{-1} Q_{t}^{0} & 0 \\
0 & 0 & E^{-1}
\end{array}\right] .
\end{aligned}
$$

The transfer function of the linear part $G_{L}(s)$ then has a block diagonal form, with the diagonal blocks given by

$$
\begin{aligned}
& G_{L}(s)_{11}=\frac{1}{s}\left(T_{2}\left(s I_{m}+M_{g}^{-1} D_{g}\right)^{-1} M_{g}^{-1} T_{2}^{t}+T_{1} D_{l}^{-1} T_{1}^{t}\right) \\
& G_{L}(s)_{22}=\left(s I_{n_{0}-m}+T_{q}^{-1}\right)^{-1} T_{q}^{-1} Q_{t}^{0} \\
& G_{L}(s)_{33}=\frac{1}{s} E^{-1} .
\end{aligned}
$$

Let

$$
\begin{aligned}
N & =\left[\begin{array}{ccc}
n_{1} I_{n-1} & 0 & 0 \\
0 & n_{3} I_{n_{0}-m} & 0 \\
0 & 0 & n_{2} I_{n_{0}}
\end{array}\right] \\
Q & =\left[\begin{array}{ccc}
q I_{n-1} & 0 & 0 \\
0 & q I_{n_{0}-m} & 0 \\
0 & 0 & q I_{n_{0}}
\end{array}\right] .
\end{aligned}
$$

By combining results from the COA analysis and from [5], we find that $G_{M}(s)=(N+Q s) G_{L}(s)$ is positive real if

$$
q \geq n_{1} \frac{M_{g_{i}}}{D_{g_{i}}} \quad i=1, \cdots, m
$$

and provided $N \geq 0$ and $Q>0$.

The rigorous development of Lyapunov functions for the MA model is given in [6]. That analysis yields

$$
\begin{aligned}
P & =\left[\begin{array}{cccc}
P_{11}(\lambda) & 0 & 0 & 0 \\
0 & 0 & 0 & 0 \\
0 & 0 & q\left(Q_{t}^{0}\right)^{-1} & 0 \\
0 & 0 & 0 & n_{2} E
\end{array}\right] \\
L & =\left[\begin{array}{cccc}
L_{11} & 0 & L_{13}(\lambda) & 0 \\
0 & 0 & 0 & 0 \\
0 & -\sqrt{2 q} T_{q}^{-\frac{1}{2}}\left(Q_{t}^{0}\right)^{-\frac{1}{2}} & 0 & 0 \\
0 & 0 & 0 & 0
\end{array}\right] \\
W & =\left[\begin{array}{cccc}
\sqrt{2 q} D_{l}^{-\frac{1}{2}} T_{1}^{t} & 0 & 0 \\
0 & \sqrt{2 q} T_{q}^{-\frac{1}{2}}\left(Q_{t}^{0}\right)^{\frac{1}{2}} & 0 \\
0 & 0 & 0 \\
0 & 0 & \sqrt{2 q} E^{-\frac{1}{2}}
\end{array}\right] .
\end{aligned}
$$

In establishing those matrices, it was convenient to restrict attention to the special case of $n_{1}=0$. (Details become rather involved for the general case of $n_{1} \neq 0$. A summary is given in [21].) Also, for consistency with the COA analysis $n_{3}=$ 0 . It turns out that solutions for $P_{11}$ are precisely the same as those outlined in [5]. In summary

$$
P_{11}(\lambda)=q M_{g}+\lambda M_{g} \underline{1} M_{g}
$$

where the scalar $\lambda$ is chosen to ensure $P_{11}(\lambda)>0$ and

$$
\begin{aligned}
- & L_{13} L_{13}^{t} \equiv Z_{l}(\lambda) \\
& =\left[-2 q D_{g}-\lambda\left(M_{g} \underline{1} D_{g}+D_{g} \underline{1} M_{g}\right)+\frac{\lambda^{2}}{2 q} d_{l} M_{g} \underline{1} M_{g}\right] \\
& \leq 0
\end{aligned}
$$

with $d_{l}=\sum_{i=1}^{n_{0}} D_{l_{i}}$. The possible choices for $\lambda$ are investigated in [5] and summarized in [14].

The Lyapunov function generated has the form given by (40)

$$
\begin{aligned}
\mathcal{V}\left(\underline{x}-\underline{x}^{s}\right) \\
=\frac{1}{2}\left(\underline{x}-\underline{x}^{s}\right)^{t}\left[\begin{array}{cccc}
P_{11}(\lambda) & 0 & 0 & 0 \\
0 & 0 & 0 & 0 \\
0 & 0 & q\left(Q_{t}^{0}\right)^{-1} & 0 \\
0 & 0 & 0 & n_{2} E
\end{array}\right]\left(\underline{x}-\underline{x}^{s}\right) \\
\\
\quad+q \int_{H^{t} \underline{x}^{s}}^{H^{t} \underline{x}}\left[\underline{\psi}(\underline{\xi})-\underline{\psi}\left(H^{t} \underline{x}^{s}\right)\right]^{t} d \underline{\xi} .
\end{aligned}
$$

From this Lyapunov function candidate, we shall now allow $E \rightarrow 0$ in (53)-(56) and (60). The resulting function

$$
\begin{aligned}
\mathcal{V}\left(\underline{x}-\underline{x}^{s}\right)= & \frac{1}{2} \underline{\omega}_{g}^{t} P_{11}(\lambda) \underline{\omega}_{g}+\frac{q}{2}\left(\underline{x}_{q}-\underline{x}_{q}^{s}\right)^{t}\left(Q_{t}^{0}\right)^{-1}\left(\underline{x}_{q}-\underline{x}_{q}^{s}\right) \\
& +q \int_{H^{t} \underline{x}^{s}}^{H^{t} \underline{x}}\left[\underline{\psi}(\underline{\xi})-\underline{\psi}\left(H^{t} \underline{x}^{s}\right)\right]^{t} d \underline{\xi}
\end{aligned}
$$

can be interpreted as a Lyapunov function candidate for the corresponding differential-algebraic system.

\section{Validity of the Lyapunov Function}

As explained in Section IV-C, it is important to check that the Lyapunov function candidate (61) is indeed a strict Lyapunov function for the power system. The first thing to check is the local positive definiteness of the function. Lemma 1 is again applicable here, with a slightly redefined $\mathcal{H}$. Similarly, Theorem 2 may be used (with minor adjustments in notation) to infer positive definiteness from the regularity of the operating point.

Analysis similar to that of Section IV-C can be used to show that $\dot{\mathcal{V}}(\underline{y}) \leq 0$ along system trajectories when $n_{1}=n_{2}=n_{3}=$ 0 . Also, a similar argument can be used to conclude that when $\dot{\mathcal{V}}(y) \equiv 0$ along a trajectory, the trajectory must be that of the system in equilibrium. LaSalle's Invariance Theorem [33] can again be used to prove asymptotic stability of the equilibrium point $\underline{x}^{s}$. 


\section{Lyapunov Function Evaluation}

From the work in [5] and in Section IV-E, the integral term of the Lyapunov function (61) evaluates to

$$
\begin{aligned}
W\left(\underline{\alpha}, \underline{x}_{q}, \underline{V}, \underline{\alpha}^{s}, \underline{x}_{q}^{s}, \underline{V}^{s}\right) \\
=-\frac{1}{2} \sum_{i=1}^{n} \sum_{j=1}^{n} B_{i j}\left(V_{i} V_{j} \cos \alpha_{i j}-V_{i}^{s} V_{j}^{s} \cos \alpha_{i j}^{s}\right) \\
\quad-\sum_{i=1}^{n-1} P_{i}^{0}\left(\alpha_{i}-\alpha_{i}^{s}\right)+\sum_{i=1}^{n_{0}-m} x_{q i} \ln \left(\frac{V_{i}}{V_{i}^{s}}\right) \\
\quad+\sum_{i=1}^{n_{0}-m} \frac{Q_{t_{i}}^{0}}{2}\left(\left(\ln \left(\frac{V_{i}}{\mu_{i}}\right)\right)^{2}-\left(\ln \left(\frac{V_{i}^{s}}{\mu_{i}}\right)\right)^{2}\right) .
\end{aligned}
$$

When evaluating (61), it is normal to set $q=1$.

It is interesting to compare this energy function with (52), the energy function for the COA model. Firstly, recall that for the COA model, $D_{g}=0$. It can be seen from (59) that with $D_{g}=0, Z_{l}(\lambda) \leq 0$ iff $\lambda=0$. From (57), $P_{11}(0)$ equals $M_{g}$.

Because of the assumption that $P_{\mathrm{COA}}=\sum_{i=1}^{n} P_{i}^{0}=0$, we have $\dot{\omega}_{0}=0$. Now $\underline{\omega}_{g}=0$ at steady-state, so by (13), $\omega_{0}=0$ at steady-state. Therefore $\omega_{0}=0$ for all time, and $\underline{\omega}_{g}=\underline{\tilde{\omega}}_{g}$. Hence $\underline{\tilde{\omega}}_{g}^{t} M_{g} \underline{\tilde{\omega}}_{g}=\underline{\omega}_{g}^{t} M_{g} \underline{\omega}_{g}$

Further, because $\sum_{i=1}^{n} P_{i}^{0}=0$, we have

$$
\begin{aligned}
\sum_{i=1}^{n} P_{i}^{0}\left(\theta_{i}-\theta_{i}^{s}\right) & =\sum_{i=1}^{n} P_{i}^{0}\left(\delta_{i}-\delta_{i}^{s}\right)-\sum_{i=1}^{n} P_{i}^{0}\left(\delta_{0}-\delta_{0}^{s}\right) \\
& =\sum_{i=1}^{n} P_{i}^{0}\left(\delta_{i}-\delta_{i}^{s}\right)
\end{aligned}
$$

and

$$
\begin{aligned}
\sum_{i=1}^{n-1} P_{i}^{0}\left(\alpha_{i}-\alpha_{i}^{s}\right) & =\sum_{i=1}^{n-1} P_{i}^{0}\left(\delta_{i}-\delta_{i}^{s}\right)-\sum_{i=1}^{n-1} P_{i}^{0}\left(\delta_{n}-\delta_{n}^{s}\right) \\
& =\sum_{i=1}^{n-1} P_{i}^{0}\left(\delta_{i}-\delta_{i}^{s}\right)+P_{n}^{0}\left(\delta_{n}-\delta_{n}^{s}\right) \\
& =\sum_{i=1}^{n} P_{i}^{0}\left(\delta_{i}-\delta_{i}^{s}\right) .
\end{aligned}
$$

So $\sum_{i=1}^{n} P_{i}^{0}\left(\theta_{i}-\theta_{i}^{s}\right)=\sum_{i=1}^{n-1} P_{i}^{0}\left(\alpha_{i}-\alpha_{i}^{s}\right)$. All other terms of the two energy functions are exactly the same.

\section{CONNECTIONS AND EXTENSIONS}

\section{A. Gradient Systems}

An approach to constructing Lyapunov functions for "quasigradient" systems was established in [8]. Assume a system has the form

$$
\underline{\dot{x}}=-A \nabla \mathcal{V}\left(\underline{x}, \underline{x}^{s}\right)
$$

where

$\mathcal{V}: R^{n} \times R^{n} \rightarrow R$ is continuously differentiable;

$A \in R^{n \times n}, \operatorname{det}(A) \neq 0$;

$\mathcal{V}\left(\underline{x}, \underline{x}^{s}\right)=0$ at $\underline{x}=\underline{x}^{s}$;

$\nabla \mathcal{V}\left(\underline{x}, \underline{x}^{s}\right)=0$ at $\underline{x}=\underline{x}^{s}$.

Let

$$
S=\left\{\underline{x} \mid \nabla \mathcal{V}\left(\underline{x}, \underline{x}^{s}\right)^{t}\left(A+A^{t}\right) \nabla \mathcal{V}\left(\underline{x}, \underline{x}^{s}\right)=0\right\}
$$

Then $\mathcal{V}\left(\underline{x}, \underline{x}^{s}\right)$ is a Lyapunov function if

1) the matrix $\left(A+A^{t}\right)$ is positive semidefinite;

2) the only complete trajectories of (63) contained in $S$ are of the form $\underline{x}(t)=\underline{x}^{e}$ for all $t \in[0, \infty)$, where $\nabla \mathcal{V}\left(\underline{x}^{e}, \underline{x}^{s}\right)=0$

3) the linearization of (63) at the equilibrium point $\underline{x}^{s}$ is strictly stable.

Consider the MA model given by (53)-(56). In that case we have

$$
\nabla \mathcal{V}=\left[\begin{array}{c}
M_{g} \underline{\omega}_{g} \\
\underline{\tilde{f}}(\underline{\alpha}, \underline{V}) \\
\left(Q_{t}^{0}\right)^{-1} \underline{x}_{q}+\underline{k}(\underline{V}) \\
\underline{g}\left(\underline{\alpha}, \underline{x}_{q}, \underline{V}\right)
\end{array}\right]
$$

and it follows that

$$
A=\left[\begin{array}{cccc}
M_{g}^{-1} D_{g} M_{g}^{-1} & M_{g}^{-1} T_{2}^{t} & 0 & 0 \\
-T_{2} M_{g}^{-1} & T_{1} D_{l}^{-1} T_{1}^{t} & 0 & 0 \\
0 & 0 & T_{q}^{-1} Q_{t}^{0} & 0 \\
0 & 0 & 0 & E^{-1}
\end{array}\right]
$$

The matrix $\left(A+A^{t}\right)$ is positive semidefinite. All the other conditions may be readily verified.

\section{B. Allowing for Voltage-Dependent Steady-State Load Characteristics}

The load model established in Section II-B, and used in the analysis so far, assumed a constant steady-state reactive power demand $Q_{s}^{0}$. However that assumption can be lifted to allow the steady-state characteristic to be voltage dependent. Let

$$
Q_{s_{i}}\left(V_{i}\right)=Q_{s_{i}}^{0} \ln \left(\frac{V_{i}}{\sigma_{i}}\right)
$$

then, if $Q_{s_{i}}^{0} \neq Q_{t_{i}}^{0}$,

$$
\begin{aligned}
& Q_{s_{i}}\left(V_{i}\right)-Q_{t_{i}}\left(V_{i}\right) \\
& \quad=\left(Q_{s_{i}}^{0}-Q_{t_{i}}^{0}\right) \ln \left(V_{i}\right)+Q_{t_{i}}^{0} \ln \left(\mu_{i}\right)-Q_{s_{i}}^{0} \ln \left(\sigma_{i}\right) \\
& \quad=\left(Q_{s_{i}}^{0}-Q_{t_{i}}^{0}\right) \ln \left(\frac{V_{i}}{\gamma_{i}}\right)
\end{aligned}
$$

where

$$
\gamma_{i}=\left(\frac{\sigma_{i}^{Q_{s_{i}}^{0}}}{\mu_{i}^{Q_{t_{i}}^{0}}}\right)^{\frac{1}{Q_{s_{i}}^{0}-Q_{t_{i}}^{0}}}
$$

Comparing (65) with (9), we see that the nonlinearity has the same general form as before. However, with the system formulated for the Popov criterion (see (34)), the new nonlinearity (65) causes a change in elements of the $G$ matrix. That alters the positive-realness condition which must be satisfied before the Popov criterion can be applied. To satisfy this positive-realness condition, it is necessary for

$$
Q_{s_{i}}^{0}<Q_{t_{i}}^{0}
$$

In the case of the first integral analysis, this condition is necessary in order to obtain a quantity whose derivative is negative definite. 


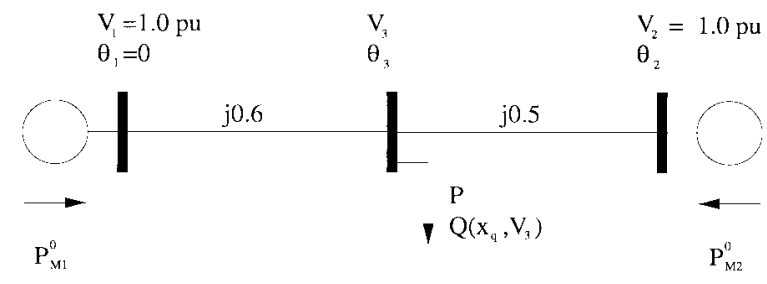

Fig. 4. Two generator, single load system.

The restriction (66) is not unrealistic. It corresponds to a load response of the general form shown in Fig. 1, where the transient load variation is greater than the long term load variation. This is the generic form of load behavior [15], [25].

The only change to the Lyapunov functions caused by voltage-dependent steady-state load characteristics is that the quadratic term relating to dynamic loads becomes

$$
\frac{1}{2}\left(\underline{x}_{q}-\underline{x}_{q}^{s}\right)^{t}\left(Q_{t}^{0}-Q_{s}^{0}\right)^{-1}\left(\underline{x}_{q}-\underline{x}_{q}^{s}\right) .
$$

\section{Comparison with Static Load Lyapunov Functions}

It is interesting to compare the Lyapunov functions developed here for dynamic loads with corresponding functions established for the static reactive power load case. We shall undertake a comparison of these functions for the MA system model. The dynamic load Lyapunov function is given by (61), with the integral term evaluated at (62). The corresponding static load Lyapunov function can be obtained from [14] and [20] as

$$
\begin{aligned}
\mathcal{V}_{s}\left(\underline{\omega}_{g}, \underline{\alpha}, \underline{V}, \underline{\alpha}^{s}, \underline{V}^{s}\right)=\frac{1}{2} \underline{\omega}_{g}^{t} P_{11}(\lambda) \underline{\omega}_{g} \\
\quad-\frac{1}{2} \sum_{i=1}^{n} \sum_{j=1}^{n} B_{i j}\left(V_{i} V_{j} \cos \alpha_{i j}-V_{i}^{s} V_{j}^{s} \cos \alpha_{i j}^{s}\right) \\
\quad-\sum_{i=1}^{n-1} P_{i}^{0}\left(\alpha_{i}-\alpha_{i}^{s}\right)+\sum_{i=1}^{n_{0}-m} \int_{V_{i}^{s}}^{V_{i}} \frac{Q_{d_{i}}\left(z_{i}\right)}{z_{i}} d z_{i}
\end{aligned}
$$

where the reactive power demand is given (statically) by $Q_{d_{i}}=Q_{d_{i}}\left(V_{i}\right)$.

Recall from (6) and (7) that for the dynamic load model

$$
Q_{d_{i}}\left(x_{q_{i}}, V_{i}\right)=x_{q_{i}}+Q_{t_{i}}^{0} \ln \left(\frac{V_{i}}{\mu_{i}}\right) .
$$

Therefore

$$
\begin{aligned}
& \int_{V_{i}^{s}}^{V_{i}} \frac{Q_{d_{i}}\left(x_{q_{i}}, z_{i}\right)}{z_{i}} d z_{i} \\
& \quad=x_{q_{i}} \ln \left(\frac{V_{i}}{V_{i}^{s}}\right)+\frac{Q_{t_{i}}^{0}}{2}\left(\left(\ln \left(\frac{V_{i}}{\mu_{i}}\right)\right)^{2}-\left(\ln \left(\frac{V_{i}^{s}}{\mu_{i}}\right)\right)^{2}\right) .
\end{aligned}
$$

So from (61), (62), the Lyapunov function for the MA model can be written

$$
\begin{aligned}
\mathcal{V}_{d}\left(\underline{\omega}_{g}, \underline{\alpha}, \underline{x}_{q}, \underline{V}, \underline{\alpha}^{s}, \underline{x}_{q}^{s}, \underline{V}^{s}\right) \\
=\frac{1}{2} \underline{\omega}_{g}^{t} P_{11}(\lambda) \underline{\omega}_{g}+\frac{1}{2}\left(\underline{x}_{q}-\underline{x}_{q}^{s}\right)^{t}\left(Q_{t}^{0}\right)^{-1}\left(\underline{x}_{q}-\underline{x}_{q}^{s}\right) \\
\quad-\frac{1}{2} \sum_{i=1}^{n} \sum_{j=1}^{n} B_{i j}\left(V_{i} V_{j} \cos \alpha_{i j}-V_{i}^{s} V_{j}^{s} \cos \alpha_{i j}^{s}\right)
\end{aligned}
$$

TABLE I

SEP POWER FLOW

\begin{tabular}{c|c|c|c|c}
\hline $\begin{array}{c}\text { Bus } \\
\text { number }\end{array}$ & $\mathrm{V}$ & $\begin{array}{c}\text { Angle } \\
\text { (deg.) }\end{array}$ & $\mathrm{P}$ & $\mathrm{Q}$ \\
\hline 1 & 1.0000 & 0.00 & -0.5 & 0.5087 \\
\hline 2 & 1.0000 & 50.90 & 0.7 & 0.6581 \\
\hline 3 & 0.7568 & 23.35 & -0.2 & -0.4000 \\
\hline
\end{tabular}

TABLE II

UEP POWER FLOW

\begin{tabular}{c|c|c|c|c}
\hline $\begin{array}{c}\text { Bus } \\
\text { number }\end{array}$ & $\mathrm{V}$ & $\begin{array}{c}\text { Angle } \\
\text { (deg.) }\end{array}$ & $\mathrm{P}$ & $\mathrm{Q}$ \\
\hline 1 & 1.0000 & 0.00 & -0.5 & 1.0881 \\
\hline 2 & 1.0000 & 90.55 & 0.7 & 1.4067 \\
\hline 3 & 0.4588 & 40.84 & -0.2 & -0.4000 \\
\hline
\end{tabular}

$$
-\sum_{i=1}^{n-1} P_{i}^{0}\left(\alpha_{i}-\alpha_{i}^{s}\right)+\sum_{i=1}^{n_{0}-m} \int_{V_{i}^{s}}^{V_{i}} \frac{Q_{d_{i}}\left(x_{q_{i}}, z_{i}\right)}{z_{i}} d z_{i} .
$$

The similarities and differences between (68) and (69) are clear.

A comparison of the equilibrium point values of $\mathcal{V}_{d}$ (for the dynamic load model) and $\mathcal{V}_{s}$ (for the static load model) also yields an interesting result. Assume all dynamic loads have a steady-state load response $Q_{s_{i}}\left(V_{i}\right)$ given by (64). To ensure the static and dynamic load models have the same equilibria, the static load model must be of the same form, i.e., $Q_{d_{i}}\left(V_{i}\right)=Q_{s_{i}}^{0} \ln \left(\frac{V_{i}}{\sigma_{i}}\right)$. Now it can be seen from (68) and (69) that the only differences between $\mathcal{V}_{d}$ and $\mathcal{V}_{s}$ lie in the terms related to the reactive power loads. Therefore consider the contribution of each (dynamic) reactive power load to the total energy $\mathcal{V}_{d}$ at an equilibrium point $\left(\underline{\alpha}^{e}, \underline{x}_{q}^{e}, \underline{V}^{e}\right)$. From (61), (62), and taking into account (67), this contribution is given by

$$
\begin{aligned}
& \mathcal{V}_{d l_{i}}\left(x_{q_{i}}^{e}, V_{i}^{e}, x_{q_{i}}^{s}, V_{i}^{s}\right) \frac{1}{2\left(Q_{t_{i}}^{0}-Q_{s_{i}}^{0}\right)}\left(x_{q_{i}}^{e}-x_{q_{i}}^{s}\right)^{2}+x_{q_{i}}^{e} \ln \left(\frac{V_{i}^{e}}{V_{i}^{s}}\right) \\
& \quad+\frac{Q_{t_{i}}^{0}}{2}\left(\left(\ln \left(\frac{V_{i}^{e}}{\mu_{i}}\right)\right)^{2}-\left(\ln \left(\frac{V_{i}^{s}}{\mu_{i}}\right)\right)^{2}\right) .
\end{aligned}
$$

Now from (5) we see that at an equilibrium point

$$
\begin{aligned}
x_{q_{i}}^{e} & =Q_{s_{i}}^{0} \ln \left(\frac{V_{i}^{e}}{\sigma_{i}}\right)-Q_{t_{i}}^{0} \ln \left(\frac{V_{i}^{e}}{\mu_{i}}\right) \\
& =\left(Q_{s_{i}}^{0}-Q_{t_{i}}^{0}\right) \ln \left(\frac{V_{i}^{e}}{\gamma_{i}}\right)
\end{aligned}
$$

and

$$
\begin{aligned}
x_{q_{i}}^{s} & =Q_{s_{i}}^{0} \ln \left(\frac{V_{i}^{s}}{\sigma_{i}}\right)-Q_{t_{i}}^{0} \ln \left(\frac{V_{i}^{s}}{\mu_{i}}\right) \\
& =\left(Q_{s_{i}}^{0}-Q_{t_{i}}^{0}\right) \ln \left(\frac{V_{i}^{s}}{\gamma_{i}}\right) .
\end{aligned}
$$

Using (71) and (72) to manipulate the first term of $\mathcal{V}_{d l_{i}}$ gives

$$
\frac{1}{2\left(Q_{t_{i}}^{0}-Q_{s_{i}}^{0}\right)}\left(x_{q_{i}}^{e}-x_{q_{i}}^{s}\right)^{2}=-\frac{1}{2} \ln \left(\frac{V_{i}^{e}}{V_{i}^{s}}\right)\left(x_{q_{i}}^{e}-x_{q_{i}}^{s}\right) .
$$




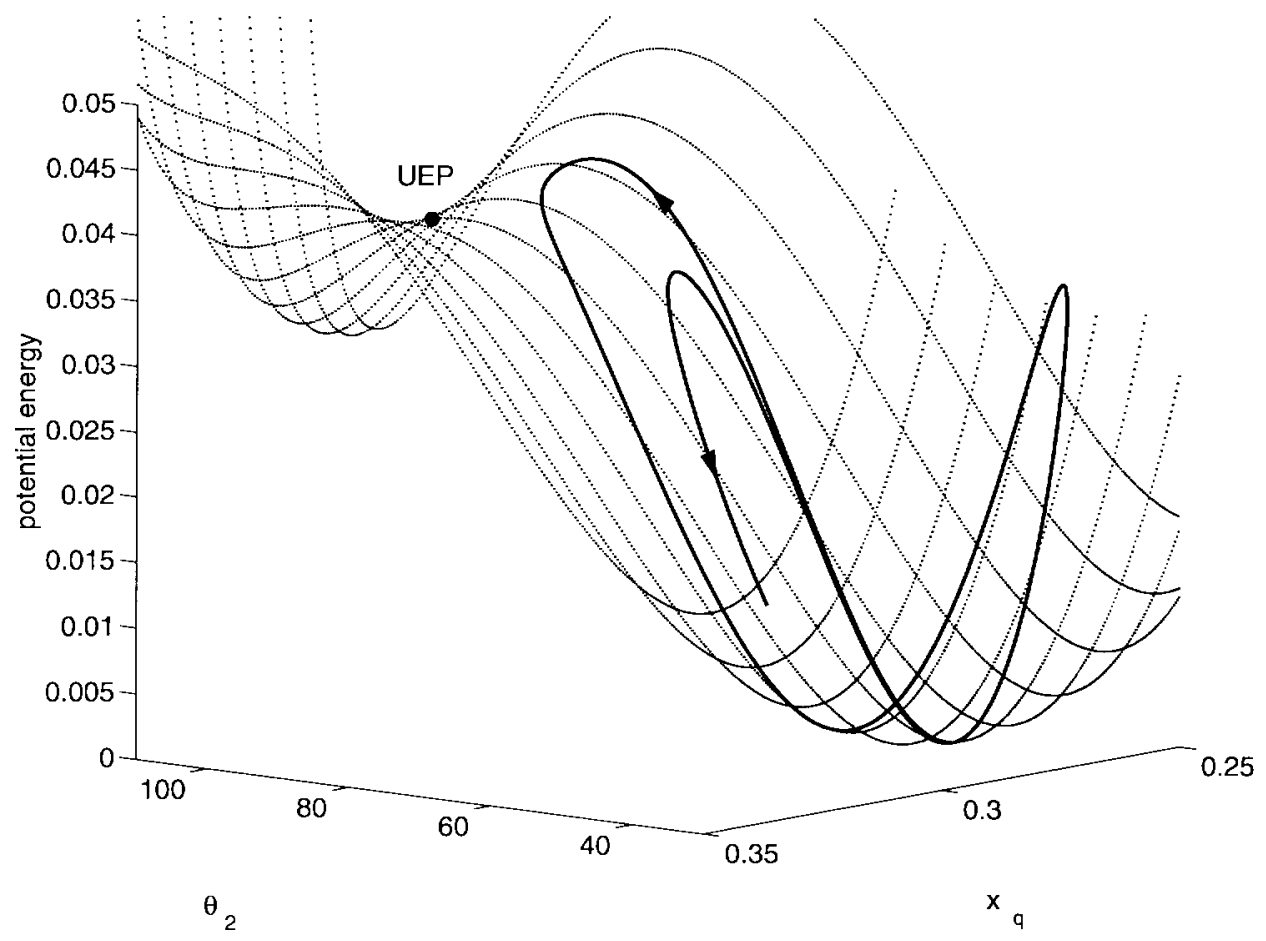

Fig. 5. Stable trajectory in the potential energy well.

Again, using (71) and (72) in the manipulation of the last term of $\mathcal{V}_{d l_{i}}$ gives

$$
\begin{aligned}
\frac{Q_{t_{i}}^{0}}{2}\left(\left(\ln \left(\frac{V_{i}^{e}}{\mu_{i}}\right)\right)^{2}-\left(\ln \left(\frac{V_{i}^{s}}{\mu_{i}}\right)\right)^{2}\right) \\
=-\frac{1}{2} \ln \left(\frac{V_{i}^{e}}{V_{i}^{s}}\right)\left(x_{q_{i}}^{e}+x_{q_{i}}^{s}\right) \\
\quad+\frac{Q_{s_{i}}^{0}}{2}\left(\left(\ln \left(\frac{V_{i}^{e}}{\sigma_{i}}\right)\right)^{2}-\left(\ln \left(\frac{V_{i}^{s}}{\sigma_{i}}\right)\right)^{2}\right) .
\end{aligned}
$$

Adding the three terms of $\mathcal{V}_{d l_{i}}$ gives

$$
\begin{aligned}
& \mathcal{V}_{d l_{i}}\left(x_{q_{i}}^{e}, V_{i}^{e}, x_{q_{i}}^{s}, V_{i}^{s}\right) \\
& =\frac{Q_{s_{i}}^{0}}{2}\left(\left(\ln \left(\frac{V_{i}^{e}}{\sigma_{i}}\right)\right)^{2}-\left(\ln \left(\frac{V_{i}^{s}}{\sigma_{i}}\right)\right)^{2}\right) .
\end{aligned}
$$

But from (68) we see that because $Q_{d_{i}}\left(V_{i}\right)=Q_{s_{i}}^{0} \ln \left(\frac{V_{i}}{\sigma_{i}}\right)$, the contribution of each (static) reactive power load to the total energy $\mathcal{V}_{s}$ is

$$
\mathcal{V}_{s l_{i}}\left(V_{i}^{e}, V_{i}^{s}\right)=\frac{Q_{s_{i}}^{0}}{2}\left(\left(\ln \left(\frac{V_{i}^{e}}{\sigma_{i}}\right)\right)^{2}-\left(\ln \left(\frac{V_{i}^{s}}{\sigma_{i}}\right)\right)^{2}\right)
$$

Hence,

$$
\mathcal{V}_{s l_{i}}\left(V_{i}^{e}, V_{i}^{s}\right)=\mathcal{V}_{d l_{i}}\left(x_{q_{i}}^{e}, V_{i}^{e}, x_{q_{i}}^{s}, V_{i}^{s}\right)
$$

so the energy at equilibria is independent of the load dynamics. This result links with ideas developed in [10].

The function (68) assumes all reactive power loads are static, whilst (69) assumes all such loads are dynamic. A Lyapunov function can easily be adapted to allow a mix of static and dynamic reactive power loads.

\section{EXAMPLES}

The aim of the examples is to explore the characteristics of the proposed Lyapunov functions. It is therefore convenient to use simple illustrative power systems. However the ideas extend naturally to large systems. Also, the examples satisfy the modeling assumptions which underlie the (strict) Lyapunov functions. Relaxation of these assumptions for practical analysis can be handled in the same way as for traditional static load Lyapunov functions [31].

\section{A. Two Machine, Single Dynamic Load System}

The two machine, single load system of Fig. 4 involves both generator and load dynamics. For a reasonably heavy load, the operating point, i.e., stable equilibrium point (SEP), is accompanied by a nearby unstable equilibrium point (UEP). For this system there is only one UEP, so it is necessarily the controlling UEP. (The potential energy of the controlling UEP gives an estimate of the critical energy $\mathcal{V}_{c}$ such that the region of state-space defined by $\left\{\underline{x}: \mathcal{V}(\underline{x})<\mathcal{V}_{c}\right\}$ provides a good estimate of the region of attraction [31].) The power flow solutions for the SEP and UEP are given in Tables I and II, respectively.

The inertia of the generator at bus 1 was set to a large value, so that the bus acted as an infinite bus. The generator at bus 2 had an inertia constant of $0.1 \mathrm{pu}$. Machine damping was set to zero to allow investigation of load damping effects. Load parameters were: $T_{q}=1.0 \mathrm{~s}, Q_{s}^{0}=0.4 \mathrm{pu}, Q_{t}^{0}=0.1 \mathrm{pu}$, $\mu=0.2$ pu.

The potential energy of the UEP was evaluated as $\mathcal{V}_{c}=$ 0.0404 pu. As discussed in Section VI-C, this energy is independent of load dynamics. 


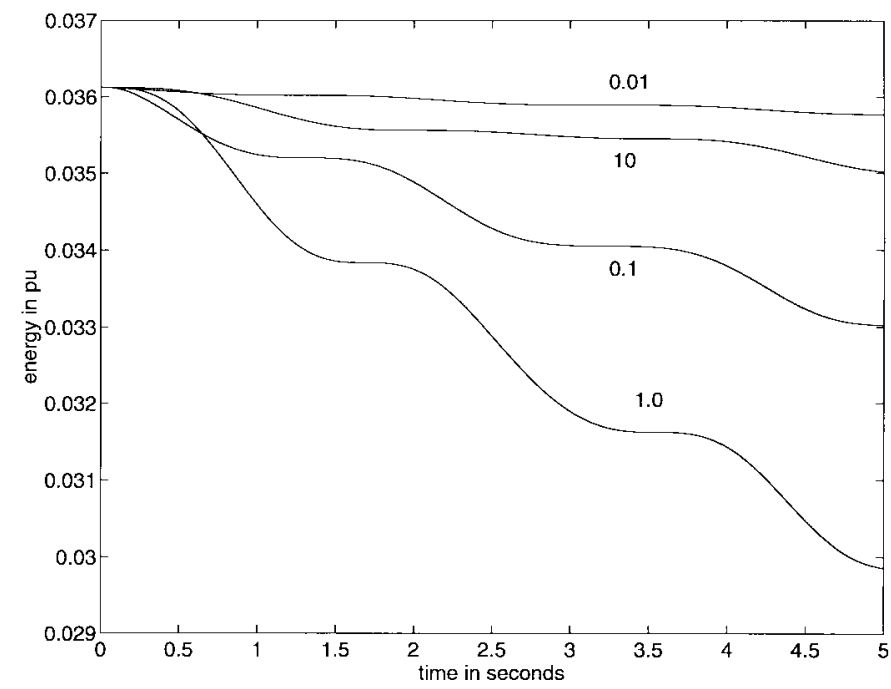

Fig. 6. Energy versus time for various values of $T_{q}$.

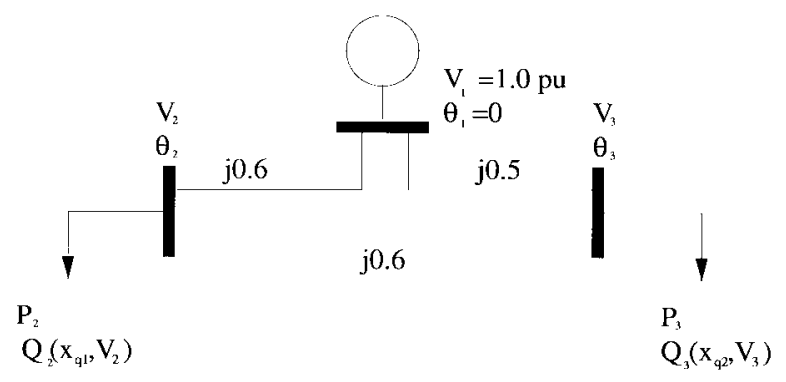

Fig. 7. Dynamic load system.

The system was faulted by opening the feeder between buses 1 and 3 . The feeder was returned to service when system energy reached the critical value $\mathcal{V}_{c}=0.0404 \mathrm{pu}$. The initial portion of the trajectory corresponding to this critically cleared case is shown in Fig. 5. This three-dimensional plot shows potential energy on the vertical axis. The potential energy well, a characteristic of energy functions, is clearly evident in this figure. Notice that the first oscillation passes close to the UEP, but the second swing shows that significant damping, due to the dynamic load, has occurred. The system is clearly stable. Delayed restoration of the feeder resulted in instability, with the trajectory exiting the potential energy well near the UEP. The mode of instability corresponded to angle separation.

It is interesting to study the effect of load time-constants upon system damping. In this case, a disturbance was simulated by assigning a nonzero value for the initial angular velocity of the generator at bus 2 . A number of simulations were conducted with various load time-constants. It can be seen that the damping was smallest for both very small and very large $T_{q}$, i.e., rapid and slow reactive power load recovery, respectively. Between these two extreme cases the damping reached a maximum. This kind of behavior was also observed in [24] for linearized systems. It can be explained using (31) which gave the rate of decrease in energy as

$$
\dot{\mathcal{V}}=-\frac{T_{q}\left(\dot{x}_{q}\right)^{2}}{Q_{t}^{0}}
$$

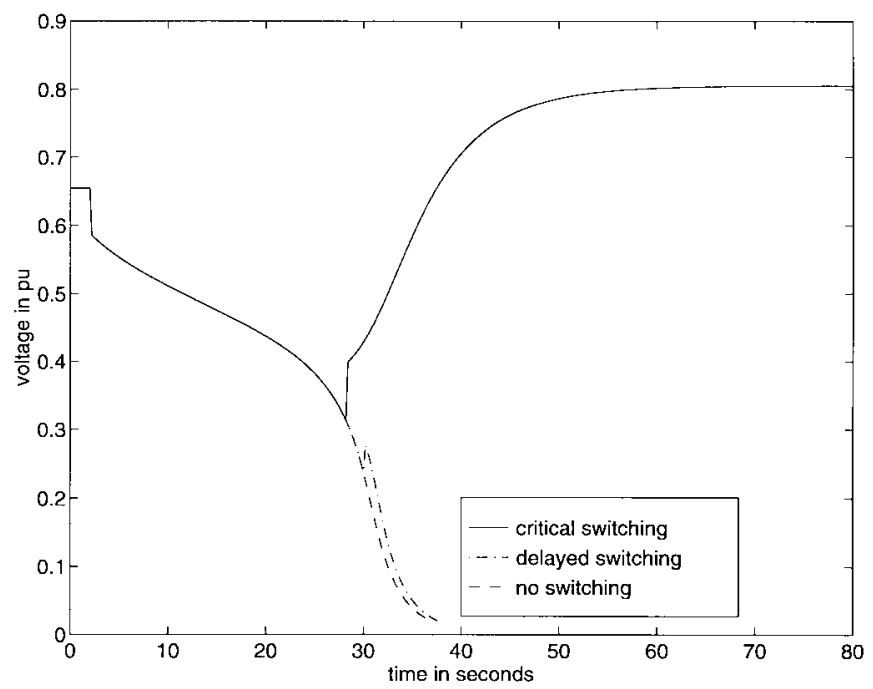

Fig. 8. Critical capacitor switching.

TABLE III

LOAD PARAMETERS

\begin{tabular}{c|c|c|c|c}
\hline Bus & & & & \\
number & $T_{q}$ & $Q_{s}^{o}$ & $Q_{t}^{o}$ & $\mu$ \\
\hline 2 & 4.0 & 0.4 & 0.3 & 0.05 \\
\hline 3 & 1.0 & 0.4 & 1.0 & 0.2 \\
\hline
\end{tabular}

When $T_{q}$ is very small it dominates the expression for $\dot{\mathcal{V}}$. The fast recovery time means that the load reactive power never deviates very far from its steady-state value $Q_{s}^{0}$. When $T_{q}$ is very large, $\left(\dot{x}_{q}\right)^{2}$ becomes very small and is the dominating factor in $\dot{\mathcal{V}}$. In this case the load behaves statically according to the logarithmic function $Q_{t}(V)$. Systems with static reactive power (in addition to the assumptions made in Section II) are known to be conservative, i.e., have zero damping. In each of the two limiting cases mentioned, conservative systems are being approached.

\section{B. Two Load System}

The system shown in Fig. 7 will be used to illustrate stability analysis of load systems. Load parameters are given in Table III. Bus 1 is an infinite bus, so there are no generator dynamics.

In this case the system was disturbed by tripping the feeder between buses 1 and 2. With no corrective switching action, the system was unstable. The voltage at bus 2 steadily declined. This is shown in Fig. 8. However stability could be maintained by switching in a shunt capacitor at bus 2 (with a susceptance of $0.4 \mathrm{pu}$ ) before the system acquired energy equal to the critical value $\mathcal{V}_{c}$ given by the controlling UEP. Time domain simulation of this stable case is also shown in Fig. 8, along with a delayed switching case. A potential energy well view of critical capacitor switching is shown in Fig. 9. This clearly illustrates the significance of the UEP energy in determining the critical switching time.

An energy-type analysis of critical capacitor switching was undertaken in [35]. This example reinforces the value of such 


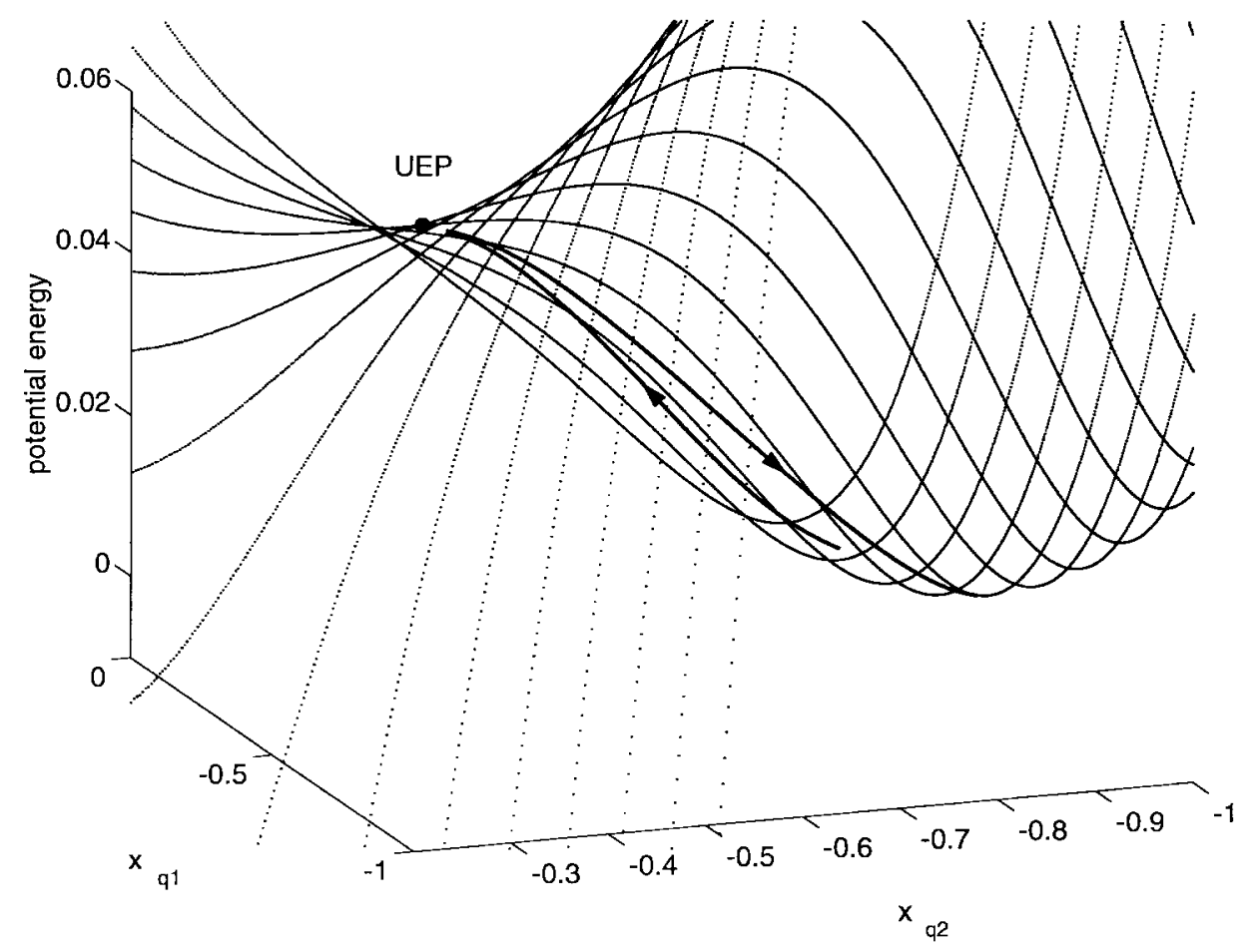

Fig. 9. Energy view of critically switched system.

an approach. However the ideas can now be justified on the basis of rigorous Lyapunov stability arguments.

\section{CONCLUSION}

This paper has developed Lyapunov functions for multimachine power systems with multiple dynamic reactive power loads. Both center of angle (COA) and machine angle (MA) model formulations have been used. The Lyapunov functions have been developed using a first integral analysis approach, and a more formalized approach based on the Popov stability criterion. These functions are extensions of similar functions developed for static reactive power loads. The paper undertakes a comparison of the static and dynamic load Lyapunov functions, and shows that they give the same value of energy at equilibria.

In order to obtain strict Lyapunov functions of the LuréPostnikov form, the transient response of the dynamic reactive power loads must have a logarithmic dependence on voltage. Real power loads must satisfy the usual constant power constraint. Relaxation of these modeling assumptions can be handled in the usual way by approximating some of the Lyapunov function terms.

The incorporation of load dynamics into a Lyapunov function has opened up a number of possibilities for energy function analysis of voltage collapse. From an analytical perspective, the Lyapunov functions provide a very useful tool for analysis of the interaction between generator angle dynamics and load voltage dynamics. Practical direct stability assessment of voltage collapse also appears promising. An example illustrates the use of a direct approach to determine the critical switching time of a capacitor such that voltage collapse is avoided.

\section{APPENDIX \\ ProOF OF THEOREM 2}

The proof of Theorem 2 is dependent upon the following definition and theorem.

Definition I.1: The inertia of a matrix $A$ is the triplet of integers $(n, z, p)$ where $n, z$, and $p$ are the number of eigenvalues of $A$ with negative, zero and positive real parts, respectively.

Theorem I.2 (Sylvester's Law of Inertia): For any nonsingular matrix $C$, the symmetric matrices $A$ and $C^{t} A C$ have the same inertia.

Proof of Theorem 2: Consider the Jacobian $J$ given at (43). It is easy to show that $\underline{g}_{x_{q}}=\underline{k}_{v}=[V]^{-1}$. So,

$$
\begin{aligned}
{\left[\begin{array}{ccc}
I & 0 & 0 \\
0 & I & 0 \\
0 & -[V]^{-1} Q_{t}^{0} & I
\end{array}\right]\left[\begin{array}{ccc}
\underline{f}_{\theta} & 0 & \underline{f}_{v} \\
0 & \left(Q_{t}^{0}\right)^{-1} & \underline{k}_{v} \\
\underline{g}_{\theta} & \underline{g}_{x_{q}} & \underline{g}_{v}
\end{array}\right] } \\
\quad \times\left[\begin{array}{ccc}
I & 0 & 0 \\
0 & I & -Q_{t}^{0}[V]^{-1} \\
0 & 0 & I
\end{array}\right] \\
=\left[\begin{array}{ccc}
\underline{f}_{\theta} & 0 & \underline{f}_{v} \\
0 & \left(Q_{t}^{0}\right)^{-1} & 0 \\
\underline{g}_{\theta} & 0 & \underline{g}_{v}-[V]^{-1} Q_{t}^{0}[V]^{-1}
\end{array}\right] \\
=J^{\prime}
\end{aligned}
$$

with all matrices evaluated at $\underline{x}=\underline{x}^{s}$. The bottom diagonal term of $J^{\prime}$ can be evaluated as

$$
\begin{aligned}
\underline{g}_{v} & -[V]^{-1} Q_{t}^{0}[V]^{-1} \\
& =\frac{\partial}{\partial V}\left([V]^{-1}\left(\underline{Q}_{l}(\underline{\theta}, \underline{V})+\underline{x}_{q}+\underline{Q}_{t}(\underline{V})\right)\right)-[V]^{-1} Q_{t}^{0}[V]^{-1}
\end{aligned}
$$




$$
\begin{aligned}
= & \frac{\partial}{\partial V}\left([V]^{-1} \underline{Q}_{l}(\underline{\theta}, \underline{V})\right)+\frac{\partial}{\partial V}\left([V]^{-1}\left(\underline{x}_{q}+\underline{Q}_{t}(\underline{V})\right)\right) \\
& \quad-[V]^{-1} Q_{t}^{0}[V]^{-1} \\
= & \frac{\partial}{\partial V}\left([V]^{-1} \underline{Q}_{l}(\underline{\theta}, \underline{V})\right)-[V]^{-1}\left[x_{q}\right][V]^{-1}+[V]^{-1} Q_{t}^{0}[V]^{-1} \\
& \quad-[V]^{-1}\left[Q_{t}(\underline{V})\right][V]^{-1}-[V]^{-1} Q_{t}^{0}[V]^{-1} \\
= & \frac{\partial}{\partial V}\left([V]^{-1} \underline{Q}_{l}(\underline{\theta}, \underline{V})\right)-[V]^{-1}\left(\left[x_{q}\right]+\left[Q_{t}(\underline{V})\right]\right)[V]^{-1}
\end{aligned}
$$

where $\left[x_{q}\right]$ and $\left[Q_{t}(\underline{V})\right]$ are diagonal matrices with elements of the corresponding vectors down the diagonal.

Because the Jacobian elements are evaluated at $\underline{x}=\underline{x}^{s}$, from (5) we have $\underline{x}_{q}+\underline{Q}_{t}(\underline{V})=\underline{Q}_{s}^{0}$. Let $Q_{s}^{0}$ be the diagonal matrix whose diagonal entries are the elements of $\underline{Q}_{S}^{0}$. Then

$$
\begin{aligned}
\underline{g}_{v}- & {[V]^{-1} Q_{t}^{0}[V]^{-1} } \\
& =\frac{\partial}{\partial V}\left([V]^{-1} \underline{Q}_{l}(\underline{\theta}, \underline{V})\right)-[V]^{-1} Q_{s}^{0}[V]^{-1} \\
& =\frac{\partial}{\partial V}\left([V]^{-1} \underline{Q}_{l}(\underline{\theta}, \underline{V})\right)+\frac{\partial}{\partial V}\left([V]^{-1} \underline{Q}_{s}^{0}\right) \\
& =\frac{\partial}{\partial V}\left([V]^{-1}\left(\underline{Q}_{l}(\underline{\theta}, \underline{V})+\underline{Q}_{s}^{0}\right)\right) .
\end{aligned}
$$

Therefore

$$
J^{\prime}=\left.\left[\begin{array}{ccc}
\underline{f}_{\theta} & 0 & \underline{f}_{v} \\
0 & \left(Q_{t}^{0}\right)^{-1} & 0 \\
\underline{g}_{\theta} & 0 & \underline{g}_{v}^{*}
\end{array}\right]\right|_{\underline{x}=\underline{x}^{s}}
$$

where $\underline{g}^{*}(\underline{\theta}, \underline{V})=[V]^{-1}\left(\underline{Q}_{l}(\underline{\theta}, \underline{V})+\underline{Q}_{s}^{0}\right)$. By Theorem I.2, $J$ and $J^{\prime}$ have the same inertia, so $J$ is positive definite iff $J^{\prime}$ is positive definite. Recall that $Q_{t}^{0}>0$ by definition. Then by rearranging rows and columns of $J^{\prime}$ it follows that $J^{\prime}$ is positive definite iff $J_{s}$, given at (44), is positive definite.

\section{REFERENCES}

[1] B. D. O. Anderson, "Stability of control systems with multiple nonlinearities," J. Franklin Inst., vol. 282, no. 3, pp. 155-160, Sept. 1966.

[2] A. R. Bergen, D. J. Hill, and C. L. DeMarco, "A Lyapunov function for multimachine power systems with generator flux decay and voltage dependent loads," Int. J. Elect. Power Energy Syst., vol. 8, no. 1, pp. 2-10, Jan. 1986.

[3] H. D. Chiang, F. F. Wu, and P. P. Varaiya, "Foundations of the potential energy boundary surface method for power system transient stability analysis," IEEE Trans. Circuits Syst., vol. 35, pp. 712-728, 1988.

[4] _ "A BCU method for direct analysis of power system transient stability," IEEE Trans. Power Syst., vol. 9, pp. 1194-1208, Aug. 1994.

[5] C. N. Chong, "Energy functions for nonlinear structure preserving models of power systems," Ph.D. dissertation, Dep. Elect. Comput. Eng., The Univ. Newcastle, Australia, 1986.

[6] R. J. Davy and I. A. Hiskens, "Lyapunov functions for multi-machine power systems with dynamic loads," Tech. Rep. EE9706, Dep. Elect. Comput. Eng., The Univ. Newcastle, Australia, Feb. 1997.

[7] C. L. DeMarco and A. R. Bergen, "Application of singular perturbation techniques to power system transient stability analysis," Memo M84/7, Electron. Res. Lab., Univ. California, Berkeley, Feb. 1984.

[8] C. L. DeMarco, "A new method of constructing Lyapunov functions for power systems," in Proc. 1988 IEEE Int. Symp. Circuits Syst., 1988, pp. $905-908$.

[9] C. L. DeMarco and C. A. Cañizares, "A vector energy function approach for security analysis of AC/DC systems," IEEE Trans. Power Syst., vol. 7, pp. 1001-1011, Aug. 1992.

[10] I. Dobson, "The irrelevance of load dynamics for the loading margin to voltage collapse and its sensitivities," in Proc. NSF/ECC Workshop on Bulk Power System Voltage Phenomena III, Davos, Switzerland, Aug. 1994, pp. 509-518.
[11] A. A. Fouad and V. Vittal, Power System Transient Stability Analysis: Using the Transient Energy Function Method. Englewood Cliffs, NJ: Prentice-Hall, 1991.

[12] D. J. Hill and A. R. Bergen, "Stability analysis of multimachine power networks with linear frequency dependent loads," IEEE Trans. Circuits Syst., vol. CAS-29, pp. 840-848, Dec. 1982.

[13] D. J. Hill and C. N. Chong, "Lyapunov functions of Luré-Postnikov form for structure preserving models of power systems," Tech. Rep. EE8529, Dep. Elect. Comput. Eng., The Univ. Newcastle, Australia, Aug. 1985.

[14] _ , "Lyapunov functions of Luré-Postnikov form for structure preserving models of power systems," Automatica, vol. 25, pp. 453-460, 1989.

[15] D. J. Hill, "Nonlinear dynamic load models with recovery for voltage stability studies," IEEE Trans. Power Syst., vol. 8, pp. 166-176, 1993.

[16] D. J. Hill and I. M. Y. Mareels, "Stability theory for differential/algebraic systems with application to power systems," IEEE Trans. Circuits Syst., vol. 37, pp. 1416-1423, Nov. 1990.

[17] D. J. Hill, I. A. Hiskens, and I. M. Y. Mareels, "Stability theory of differential/algebraic models of power systems," in Proc. Eng. Sci. Indian Acad. Sci., Special Issue on Nonlinear Dynamics: Applications in Power Systems, Sept. 1993, vol. 18, part 5, pp. 731-747.

[18] D. J. Hill, I. A. Hiskens, and D. Popović, "Load recovery in voltage stability analysis and control," in Proc. NSF/ECC Workshop on Bulk Power System Voltage Phenomena III, Davos, Switzerland, Aug. 1994, pp. 579-595.

[19] D. J. Hill and I. A. Hiskens, "Dynamic analysis of voltage collapse in power systems," in Systems and Control Theory for Power Systems, J. H. Chow, P. V. Kokotovic, and R. J. Thomas, Eds. New York: Springer-Verlag, 1995, pp. 157-172.

[20] I. A. Hiskens and D. J. Hill, "Energy functions, transient stability and voltage behavior in power systems with nonlinear loads," IEEE Trans. Power Syst., vol. 4, pp. 1525-1533, Nov. 1989.

[21] _ "Failure modes of a collapsing power system," in Proc. NSF/ECC Workshop on Bulk Power System Voltage Phenomena II, Deep Creek Lake, MD, Aug. 1991, pp. 53-64.

[22] _ "Incorporation of SVC's into energy function methods," IEEE Trans. Power Syst., vol. 7, pp. 133-140, Feb. 1992.

[23] _ "Modeling of dynamic load behavior," in Proc. NSF/ECC Workshop on Bulk Power System Voltage Phenomena III, Davos, Switzerland, Aug. 1994, pp. 501-508.

[24] I. A. Hiskens and J. V. Milanović, "Load modeling in studies of power system damping," IEEE Trans. Power Syst., vol. 10, pp. 1781-1788, Nov. 1995.

[25] D. Karlsson and D. J. Hill, "Modeling and identification of nonlinear dynamic loads in power systems," IEEE Trans. Power Syst., vol. 9, pp. 157-166, Feb. 1994.

[26] N. Narasimhamurthi and M. T. Musavi, "A general energy function for transient stability analysis of power systems," IEEE Trans. Circuits Syst., vol. CAS-31, pp. 637-645, July 1984.

[27] K. R. Padiyar and H. S. Y. Sastry, "Topological energy function analysis of stability of power systems," Int. J. Elect. Power Energy Syst., vol. 9, no. 1, pp. 9-16, Jan. 1987.

[28] K. R. Padiyar and K. K. Ghosh, "Direct stability evaluation of power systems with detailed generator models using structure preserving energy functions," Int. J. Elect. Power Energy Syst., vol. 11, no. 1, pp. 47-56, Jan. 1989.

[29] K. R. Padiyar and V. Immanuel, "Modeling of SVC for stability evaluation using structure preserving energy function," Int. J. Elect. Power Energy Syst., vol. 16, no. 5, pp. 339-348, 1994.

[30] M. A. Pai, Power System Stability. Amsterdam, The Netherlands: North-Holland, 1981

[31] M. A. Pai, Energy Function Analysis for Power System Stability. Boston, MA: Kluwer Academic, 1989.

[32] T. Van Cutsem and M. Ribbens-Pavella, "Structure preserving direct methods for transient stability analysis of power systems," in Proc. 24th IEEE Conf. Decision Contr., Ft. Lauderdale, FL, Dec. 1985, pp. $70-76$.

[33] M. Vidyasagar, Nonlinear Systems Analysis, 2nd ed. Englewood Cliffs, NJ: Prentice-Hall, 1993.

[34] J. L. Willems, "Optimum Lyapunov functions and stability regions for multimachine power systems," Proc. Inst. Elect. Eng., vol. 117, pp. 573-577, 1970.

[35] W. Xu and Y. Mansour, "Voltage stability analysis using generic dynamic load models," IEEE Trans. Power Syst., vol. 9, pp. 479-493, Feb. 1994. 


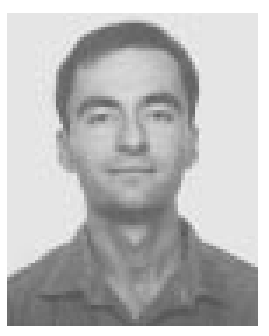

Robert J. Davy received the B.E. degree in electrical engineering in 1993, and the M.E. degree in 1997, both from the University of Newcastle, Australia.

$\mathrm{He}$ is currently working in the field of process control engineering. His research interests have included the stability of nonlinear dynamical systems, with application to power systems.

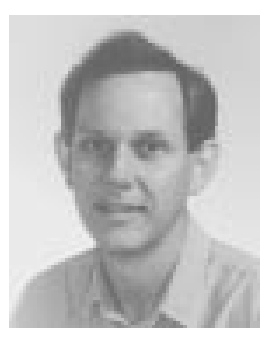

Ian A. Hiskens (S'77-M'80-SM'96) received the B.Eng. (Elec.) degree and the B.App.Sc. (Math.) degree from the Capricornia Institute of Advanced Education, Rockhampton, Australia, in 1980 and 1983, respectively. He received the Ph.D. degree from the University of Newcastle, Australia, in 1990.

From 1980 to 1992 he was with the Queensland Electricity Supply Industry. He is currently a Senior Lecturer with the Department of Electrical and Computer Engineering, University of Newcastle. His major research interests lie in the area of power system analysis, in particular, system dynamics and control, security, and numerical techniques. Other research interests include nonlinear systems and control. 